\title{
Plexin-B2, But Not Plexin-B1, Critically Modulates Neuronal Migration and Patterning of the Developing Nervous System In Vivo
}

\author{
Suhua Deng, ${ }^{1}$ Alexandra Hirschberg, ${ }^{1}$ Thomas Worzfeld, ${ }^{1}$ Junia Y. Penachioni, ${ }^{2}$ Alexander Korostylev, ${ }^{1}$ \\ Jakub M. Swiercz, ${ }^{1}$ Peter Vodrazka, ${ }^{1}$ Olivier Mauti, ${ }^{3}$ Esther T. Stoeckli, ${ }^{3}$ Luca Tamagnone, ${ }^{2}$ Stefan Offermanns, ${ }^{1}$ and \\ Rohini Kuner ${ }^{1}$ \\ ${ }^{1}$ Institute of Pharmacology, University of Heidelberg, 69120 Heidelberg, Germany, ${ }^{2}$ Institute for Cancer Research and Treatment, University of Torino \\ School of Medicine, 10060 Candiolo, Torino, Italy, and ${ }^{3}$ Institute of Zoology, University of Zürich, CH-8057 Zürich, Switzerland
}

\begin{abstract}
Semaphorins and their receptors, plexins, have emerged as important cellular cues regulating key developmental processes. B-type plexins directly regulate the actin cytoskeleton in a variety of cell types. Recently, B-type plexins have been shown to be expressed in striking patterns in the nervous system over critical developmental windows. However, in contrast to the well characterized plexin-A family, the functional role of plexin-B proteins in neural development and organogenesis in vertebrates in vivo is not known. Here, we have elucidated the functional contribution of the two neuronally expressed plexin-B proteins, Plexin-B1 or Plexin-B2, toward the development of the peripheral nervous system and the CNS by generating and analyzing constitutive knock-out mice. The development of the nervous system was found to be normal in mice lacking Plexin-B1, whereas mice lacking Plexin-B2 demonstrated defects in closure of the neural tube and a conspicuous disorganization of the embryonic brain. After analyzing mutant mice, which bypassed neural tube defects, we observed a key requirement for Plexin-B2 in proliferation and migration of granule cell precursors in the developing dentate gyrus, olfactory bulb, and cerebellum. Furthermore, we identified semaphorin 4C as a high-affinity ligand for Plexin-B2 in binding and functional assays. Semaphorin 4C stimulated activation of ErbB-2 and RhoA via Plexin-B2 and enhanced proliferation and migration of granule cell precursors. Semaphorin 4C-induced proliferation of ventricular zone neuroblasts was abrogated in mice lacking Plexin-B2. These genetic and functional analyses reveal a key requirement for Plexin-B2, but not Plexin-B1, in patterning of the vertebrate nervous system in vivo.
\end{abstract}

Key words: semaphorin; Rho GTPases; knock-out mice; granule cell; dentate gyrus; cerebellum

\section{Introduction}

Semaphorins constitute an important family of secreted or membrane-bound cues. Although semaphorins were initially described to function as important axon-guidance molecules (Raper, 2000; Liu and Strittmatter, 2001; Fiore and Puschel, 2003), several recent studies have established them as key regulators of organogenesis, angiogenesis, invasive growth, and apoptosis (Giordano et al., 2002; Conrotto et al., 2005; Gu et al., 2005; Neufeld et al., 2005). The cellular effects of semaphorins are achieved via activation of transmembrane receptors called plexins and their coreceptors, the neuropilins.

\footnotetext{
Received Dec. 13, 2006; revised April 4, 2007; accepted April 30, 2007.

This work was supported by SFB488 grants from the Deutsche Forschunggemeinschaft (DFG) to R.K. and S.0., grants from the Italian Ministry of Research (MIUR-PRIN2005), and the Italian Association for Cancer Research to L.T., a Graduate College 791 fellowship from the DFG to S.D., and postdoctoral program fellowships from the Medical Faculty of the University of Heidelberg to T.W. and J.M.S. We thank Steven Strittmatter for providing the Sema4C-AP CDNA, Shinobu Inagaki for providing Plexin-B2 CDNA, Iris Hack for help with explant cultures, and Hans-Joseph Wrede for technical support.

Correspondence should be addressed to either Stefan Offermanns or Rohini Kuner, Institute of Pharmacology, University of Heidelberg, Im Neuenheimer Feld 366, 69120 Heidelberg, Germany, E-mail: stefan.offermanns@pharma.uni-heidelberg.de or rohini.kuner@pharma.uni-heidelberg.de.

D0I:10.1523/JNEUROSC1.5381-06.2007

Copyright $\odot 2007$ Society for Neuroscience $\quad$ 0270-6474/07/276333-15\$15.00/0
}

In contrast to the well characterized plexin-A family, very little was known until recently about the ligands, the expression, and the cellular effectors of the plexin-B family proteins. Plexin-B1, the prototypic member, is known to bind semaphorin $4 \mathrm{D}$ (Sema4D) in its transmembrane form as well as its soluble N-terminal, proteolysed product (Tamagnone et al., 1999). Although recent studies have shown that Sema4D can bind Plexin-B2 in vitro (Masuda et al., 2004), whether Sema4D can serve as a natural ligand for Plexin-B2 in vivo is inconclusive. Another semaphorin, Sema5A, was recently shown to bind Plexin-B3 in vitro (Artigiani et al., 2004).

We and others have initially described the functional interaction of plexin-B family members with a family of neuronally expressed Rho guanine nucleotide exchange factors (RhoGEFs), PDZRhoGEF and LARG (Aurandt et al., 2002; Hirotani et al., 2002; Perrot et al., 2002; Swiercz et al., 2002), thereby providing the missing link between activation of plexin-B and the Rho GTPase, RhoA, which is known to be a critical regulator of neuronal polarity, migration, and morphogenesis in developing neurons (Fiore and Puschel, 2003; Guan and Rao, 2003; Govek et al., 2005; Negishi et al., 2005). Plexin-B1 also physically associates with the receptor tyrosine kinases Met and ErbB-2, which are activated 
indirectly during binding of Sema4D to Plexin-B1 (Giordano et al., 2002; Swiercz et al., 2004). Moreover, Sema4D/Plexin-B1 can inhibit the activity of R-Ras and sequester activated Rac (Vikis et al., 2000; Oinuma et al., 2004). Consistent with a role for plexinB-dependent signaling pathways in development of the nervous system, we have previously shown that plexin-B family members demonstrate very striking and nonredundant patterns of expression during critical time windows of neural development in mice (Worzfeld et al., 2004).

To date, however, very little is known about the significance of plexin- $\mathrm{B}$ family proteins in shaping the architecture and the function of the developing nervous system in vivo. The only in vivo studies on plexin-B functions in neural development thus far have come from Drosophila, in which aberrations in nerve connectivity were found in plexin-B mutants (Hu et al., 2001; Ayoob et al., 2006). To elucidate the functional significance of plexin-B family proteins in various aspects of vertebrate neural development, we generated genetically modified mice constitutively lacking Plexin-B1 or Plexin-B2. We observed that a loss of Plexin-B1 expression in mice does not significantly impact the development of the nervous system. In contrast, Plexin-B2 is critically required for several developmental processes in vivo, including proliferation, migration, and pattern formation in the mouse forebrain and the cerebellum.

\section{Materials and Methods}

Generation of mice lacking Plexin-B1 or Plexin-B2. The gene structure described here and in the knock-out strategy in Figures 2 and 3 is based on Vega annotations in the Ensembl database ( plxnb1 gene, transcript number OTTMUST00000049801; plxnb2 gene, transcript number OTTMUST00000049692). We sought to delete portions of the plxnb genes, which either encode the transmembrane domain of the respective Plexin-B proteins or lie upstream thereof, thereby generating an early translational stop via a frameshift mutation. The transmembrane domains of Plexin-B1 and Plexin-B2 are encoded by exon 23 of the mouse plxnb1 gene and exon 22 the plxnb2 gene, respectively. A neo/TK selection cassette flanked by loxP sites was introduced into intron 12 of the mouse plxnbl gene, and an additional loxP site was introduced into intron 17 in embryonic stem (ES) cells (a strategy view is shown in Fig. $2 A$, Southern blot analysis is shown in $B$ ). Mice obtained from a targeted ES cell clone were mated with mice that express the Cre recombinase at the zygote stage resulting in recombination in all cells (Lakso et al., 1996). The resulting progeny lacked exons 13-16 of the plxnb1 gene (Southern blot analysis shown in Fig. 2C), thereby leading to a frameshift and a translational stop after exon 12. In mice homozygous for this targeted deletion ( $p l x n b 1^{-/-}$), the plexin-B1 mRNA transcript could not be detected via Northern blot analysis (Fig. 2D), in situ hybridization (supplemental Fig. 1, available at www.jneurosci.org as supplemental material), or reverse transcription (RT)-PCR (data not shown).

A neo/TK selection cassette flanked by loxP sites was introduced into intron 18 of the mouse plxnb2 gene, and an additional loxP site was introduced into intron 23 in ES cells (a strategy view is shown in Fig. $3 A$, Southern blot analysis is shown in $B$ ). After transfection of a plasmid expressing Cre recombinase in targeted ES cells, correctly recombined clones were used to derive mice lacking exons 19-23 of the plxnb2 gene (Southern blot analysis in Fig. 3C), leading to a frameshift, a premature translational stop after exon 18, and loss of the transmembrane domain (exon 22). In embryos homozygous for this targeted deletion ( $p l \times n b 2^{-1}$ -), the plexin-B2 mRNA transcript could not be detected via Northern blot analysis (Fig. 3D), in situ hybridization (supplemental Fig. 1, available at www.jneurosci.org as supplemental material), or RT-PCR (data not shown). Plxnb2 $2^{-1-}$ embryos did not show any changes in the expression levels of plexin-B1 transcripts (data not shown).

Here, plexin-B1, plexin-B2, plexin-B2, Sema4d, and Sema4c refer to mRNA transcripts, and Plexin-B1, Plexin-B2, Plexin-B3, Sema4D, and Sema4C refer to protein.
Antibodies, Western blotting, and immunohistochemistry. The following antibodies and reagents were used: rabbit anti-substance P (1:200; Millipore, Billerica, MA), biotinylated-IsolectinB4 (1:200; Vector Laboratories, Burlingame, CA), goat anti-NeuroD (1:100; Santa Cruz Biotechnology, Santa Cruz, CA), mouse anti-calbindin (1:2000; Millipore), mouse anti-neuronal-specific nuclear protein (NeuN; 1:2000; Chemicon), rabbit anti-glial fibrillary acidic protein (GFAP; 1:500; Dako, High Wycombe, UK), rabbit anti-tyrosine hydroxylase (1:500; Chemicon), rabbit anti-calretinin (1:600; Chemicon), mouse anti-GAP43 (1:2000; Sigma, St. Louis, MO), mouse anti-polysialylated neural cell adhesion molecule (PSA-NCAM; 1:300; Chemicon), mouse anti-GAD67 (1:1500; Chemicon), mouse anti- $\beta$ III-tubulin (1:100; Chemicon), rabbit anti-Prox-1 (1:100; Chemicon), rat anti-Ki-67 (1:25; Dako), mouse anti-5-bromo-2deoxyuridine (BrdU; 1:500; Sigma), mouse anti-CNPase (1:500; Chemicon), rabbit anti-TAG-1 (1:1000; Esther Stoeckli, University of Zurich, Zurich, Switzerland), mouse anti-vesicular stomatitis virus (VSV; Sigma), mouse anti-hemagglutinin (HA) and goat anti- $\beta$-actin (Santa Cruz Biotechnology), mouse anti-plexin-B1 (EC6.9 and IC2) (Barberis et al., 2004), anti-Plexin-B2 (1-16; 1: 100; Santa Cruz Biotechnology), anti-phospho-ErbB-2 (Tyr 1248; 1:400; Cell Signaling Technology, Beverly, MA), and anti-ErbB-2 (1:300, Cell Signaling Technology). Western blotting was performed according to standard laboratory protocols. Mice were perfused with $4 \%$ paraformaldehyde (PFA) and spinal cords, and brains were extracted and postfixed overnight in 4\% PFA. Immunohistochemistry was performed on vibratome sections $(50 \mu \mathrm{m})$ or cryosections $(20 \mu \mathrm{m})$ using standard reagents and protocols (Vector Laboratories).

Whole-mount immunostaining of spinal and cranial nerves. After dissection and fixation, embryos were rinsed well in PBS before being transferred to 24-well plates. They were permeabilized with $1 \%$ Triton X-100 in PBS for $1 \mathrm{~h}$ at room temperature. After incubation in $20 \mathrm{~mm}$ lysine in 0.1 m sodium phosphate buffer, $\mathrm{pH}$ 7.4, for $1 \mathrm{~h}$, they were rinsed in PBS followed by a preincubation step in blocking buffer (10\% FCS in PBS) for at least $2 \mathrm{~h}$ at room temperature. For staining, a rabbit antineurofilament $\mathrm{M}$ antibody was diluted in blocking buffer (final dilution 1:250; incubation at $4^{\circ} \mathrm{C}$ for $48 \mathrm{~h}$; Abcam, Cambridge, UK). After washing the embryos thoroughly in PBS overnight at $4^{\circ} \mathrm{C}$, they were incubated in blocking buffer for at least $2 \mathrm{~h}$ before adding the secondary antibody (donkey anti-rabbit IgG cyanine $3 ; 1: 250$ ) overnight at $4^{\circ} \mathrm{C}$. After thoroughly rinsing the embryos, they were dehydrated in a graded series of methanol $(25,50,75$, and $100 \%$, two times for $10 \mathrm{~min}$ each). They were transferred to a mixture of benzyl benzoate/benzyl alcohol (2:1) and analyzed with fluorescent optics.

Northern blot analysis. Primers used for amplification of Plexin-B1 and Plexin-B2 mouse cDNA fragments, which served as probes for Northern blot analysis, were 5'-GGA GTG TGT TCT CTG TGG AG-3' (sense probe for Plexin-B1), 5' -TCG GAA GTG ACG TCC TCA TC-3' (antisense probe for Plexin-B1), 5' -CAA GCG GCG GCA GAA GCG AGA-3' (sense probe for Plexin-B2), and 5'-ATC TGC TGT AGG CGG AAG GCC AG-3' (antisense probe for Plexin-B2). Total RNA (10 $\mu \mathrm{g})$ derived from adult mouse brains or mouse embryos at embryonic stage 17.5 was resolved on $1 \%$ denaturing agarose gels and transferred onto nylon membranes that were hybridized with a $\left[{ }^{32} \mathrm{P}\right]$ dATP-labeled Plexin-B1or Plexin-B2-cDNA probes, respectively (specific activity, $>1 \times 10^{9}$ $\mathrm{cpm} / \mu \mathrm{g}$ ) for $3 \mathrm{~h}$ at $68^{\circ} \mathrm{C}$ in Quikhyb Solution (Stratagene, La Jolla, CA). After washing twice with $0.1 \times \mathrm{SSC} / 0.1 \%$ SDS at $68^{\circ} \mathrm{C}$ for $30 \mathrm{~min}$, the hydridized probe was visualized by autoradiography. Equal loading of the lanes was confirmed by comparing the intensities of the $18 \mathrm{~S}$ ribosomal RNA bands on the denaturing agarose gel.

In situ hybridization. In situ hybridization experiments were done on cryostat sections using digoxigenin-labeled nonradioactive probes exactly as described previously (Worzfeld et al., 2004). The generation of the riboprobes recognizing Sema4d, plexin-B1, and plexin-B2 has been described in detail previously (Worzfeld et al., 2004). A 2-kb-long riboprobe corresponding to the $3^{\prime}$ untranslated region of the mouse Sema4C cDNA was generated via in vitro transcription from a template generated via PCR using the primers (1) forward (5'-TGC CAC CTA TAC AGT GTT GTT C-3') and (2) reverse (5'-GGG GAC ACA ATA AGT TAC ACT G-3'). Blue-purple color was judged to constitute positive hybrid- 
ization signals. Hybridizations with sense riboprobes were included in all experiment as controls for nonspecific background signals.

Explant cultures. Cultures of subventricular zone (SVZ) explants were performed as described previously (Hack et al., 2002). Brains of postnatal day 5 (P5) mice were sectioned, the SVZ was dissected out from the lateral wall of the anterior horn of the lateral ventricle in vibratome-cut sections, and 100-300 $\mu \mathrm{m}$ diameter explants were prepared, mixed with Matrigel (BD Biosciences, San Jose, CA), and cultured in 24-well plates. After polymerization for $15 \mathrm{~min}$, the gel was covered with $500 \mu \mathrm{l}$ of serum-free medium containing B-27 supplement (Invitrogen, Carlsbad, $\mathrm{CA}$ ) and alkaline phosphatase (AP)-tagged Sema4D (Sema4D-AP) medium or control medium from human embryonic kidney (HEK) cells for $48 \mathrm{~h}$. Cultures were maintained in a humidified, $5 \% \mathrm{CO}_{2}, 37^{\circ} \mathrm{C}$ incubator. Cultures were fixed for $20 \mathrm{~min}$, blocked for $30 \mathrm{~min}$, and then stained with antibody against PSA-NCAM (1:100; Chemicon).

For external granular layer (EGL) microexplant culture (Nagata and Nakatsuji, 1990), cerebella from P5 pups were sectioned, and rectangular pieces $(250-400 \mu \mathrm{m})$ were dissected out from EGL. Microexplants were placed on the poly-L-lysine/laminin-coated glass coverslips with $50 \mu \mathrm{l}$ of the culture medium. Two hours later, cultures were overlaid with $450 \mu \mathrm{l}$ of the culture medium containing B-27 supplement (Invitrogen) and Sema4D-AP medium or control medium from HEK cells for $48 \mathrm{~h}$ in a humidified, $5 \% \mathrm{CO}_{2}$ atmosphere at $37^{\circ} \mathrm{C}$. Cultures were fixed for $20 \mathrm{~min}$, blocked for $30 \mathrm{~min}$ with goat serum and then stained with $4^{\prime}, 6^{\prime}$ diamidino-2-phenylindole dihydrochloride (DAPI) and TRITCconjugated phalloidin for $1 \mathrm{~h}$ at room temperature.

Slice culture assay. Coronal slices (500 $\mu \mathrm{m}$ thickness) were prepared from the cerebella of P5 plxnb1 $1^{+/-}$and $p l x n b 1^{-1-}$ mouse pups. Slices were transferred to Millicell (Millipore, Billerica, MA) membrane inserts $(0.4 \mu \mathrm{m})$ and placed in $6 \mathrm{~cm}$ culture dishes with $1.5 \mathrm{ml}$ of slice culture media, consisting of $50 \%$ basal medium with Eagle's salts, $25 \%$ heatinactivated horse serum, 25\% HBSS, $45 \mu \mathrm{g} / \mathrm{ml}$ D-glucose, $0.1 \mathrm{~mm}$ $\mathrm{L}$-glutamine, and $100 \% \mathrm{U} / \mathrm{ml}$ streptomycin/penicillin. The upper surfaces of the slices were covered by one drop of culture medium. Slices were incubated at $37^{\circ} \mathrm{C}$ with $5 \% \mathrm{CO}_{2}$ for $24,48,72$, and $96 \mathrm{~h}$. The medium was changed every other day. To observe granule cell migration, organotypic slices were sectioned with $20 \mu \mathrm{m}$ thickness on cryostat and followed by immunohistochemistry.

Boyden chamber migration assay. Cerebellar granule cell preparation and Boyden chamber assay was done as described previously (Klein et al., 2001). Briefly, cerebellar granule cells were dissociated from P5 plxnb1 $1^{+-}$and $p l x n b 1^{-1-}$ mice. Laminin-coated polyvinylcarbonatefree membranes (Neuroprobe, Gaithersburg, MD) with $8 \mu \mathrm{m}$ pore size were used to evaluate the in vitro migration of granule cells. Approximately $2 \times 10^{6}$ cells $/ \mathrm{ml}$ in $50 \mu \mathrm{l}$ of serum-free DMEM were added to the upper chambers. Lower chambers were filled in $27 \mu \mathrm{l}$ of DMEM, DMEM with purified Sema4D $(8 \mu \mathrm{g} / \mathrm{ml})$, or DMEM with human stromal cellderived factor $1 \alpha($ SDF- $1 \alpha)(1 \mathrm{mg} / \mathrm{ml})$. In some experiments, medium of HEK293 cells that secreted AP-tagged Sema4C was concentrated 100fold and diluted to a final threefold concentration $(3 \times)$ in DMEM used for the migration assays. In these experiments, medium of HEK293 cells that expressed an empty AP plasmid was used as a control at a concentration similar to that described above. Chambers were incubated overnight at $37^{\circ} \mathrm{C}$ in $5 \% \mathrm{CO}_{2}$. Cells in the upper surface of membranes were scraped, and the migrated cells in the lower surface were fixed and stained with Dif-Quik cell fixation and staining kit (Baxter, Deerfield, IL). Four to five randomly chosen fields at high magnification were counted in each well. Experiments were performed at least four times under each condition.

Adhesion assay. Cells were counted, spun, and resuspended in serumfree medium, containing $0.2 \%$ BSA. Cells $\left(6 \times 10^{4}\right)$ were seeded in 96-well plates, previously coated with adhesive substrates: $10 \mu \mathrm{g} / \mathrm{ml}$ laminin, $10 \mu \mathrm{g} / \mathrm{ml}$ fibronectin, or $1 \mu \mathrm{g} / \mathrm{ml}$ poly-L-lysine, and blocked with $0.2 \% \mathrm{BSA}$. Incubation was done at $37^{\circ} \mathrm{C}$ in a $5 \% \mathrm{CO}_{2}$ atmosphere in serum-free DMEM containing purified Sema4D ( $5 \mu \mathrm{g} / \mathrm{ml})$, Sema4D-AP concentrated medium $(3 \times)$, Sema4C-AP concentrated medium $(3 \times)$, or mock concentrated medium $(3 \times)$ from HEK 293 cells. At $2 \mathrm{~h}$, the wells were washed twice with PBS, and the adherent cells were fixed and subsequently stained with crystal violet. To quantify cell adhesion, the dye was then eluted from adherent cells with $10 \%$ acetic acid, and the absorbance measured at $595 \mathrm{~nm}$ in a microplate reader.

In vitro BrdU-incorporation assay. For BrdU-incorporation assays on cerebellar granule cell precursors (GCPs) isolated from postnatal cerebellum, $3 \times 10^{5}$ cells were seeded in 24-well plates on coverslips coated with $1 \mu \mathrm{g} / \mathrm{ml}$ poly-L-lysine. After $72 \mathrm{~h}$, cells were treated with Sema4D-AP concentrated medium $(3 \times)$, Sema4C-AP concentrated medium $(3 \times)$, mock concentrated medium $(3 \times)$ or purified Sema4D (5 $\mu \mathrm{g} / \mathrm{ml})$ plus BrdU (10 ng/ml) for $4 \mathrm{~h}$. The cells were fixed in $4 \%$ ice-cold $\mathrm{PFA}$, washed in PBS, denaturated with $4 \% \mathrm{HCl}$, and then neutralized with sodium borate buffer, $\mathrm{pH} 8.5$, and washed three times with PBS. After blocking in 10\% NGS for $30 \mathrm{~min}$ at room temperature, cells were immunostained with an anti-BrdU antibody (1:200; Sigma). For performing BrdU-incorporation assays on dissociated ventricular zone (VZ) cells, the cerebral hemispheres were dissected out embryonic day 15 (E15) embryos. After removing the meninges, the VZ (possibly with components of the SVZ) was manually dissected from the lateral cortices and dissociated into single-cell suspensions using trypsin digest described previously (Behar et al., 1998). Dissociated cells were resuspended in plating medium without serum and $4.5 \times 10^{4}$ cells were seeded in 48-well plates onto coverslips precoated with $1 \mu \mathrm{g} / \mathrm{ml}$ poly-Dlysine, where they were allowed to adhere for $24 \mathrm{~h}$ at $37^{\circ} \mathrm{C}$. Cells were subsequently treated with Sema4C-AP concentrated medium $(3 \times)$, mock concentrated medium $(3 \times)$, or epidermal growth factor $(E G F)(10$ $\mathrm{ng} / \mathrm{ml}$ ) together with $50 \mathrm{ng} / \mathrm{ml} \mathrm{BrdU}$ for $4 \mathrm{~h}$ and processed as described above.

In vivo BrdU-incorporation assay. Littermates of $\mathrm{P} 5$ pups were injected intraperitoneally with $100 \mathrm{mg} / \mathrm{kg}$ BrdU and were killed 1 and $28 \mathrm{~h}$ after injection. Pregnant female mice were injected intraperitoneally with 300 $\mathrm{mg} / \mathrm{kg}$ BrdU on E16.5, and embryos were harvested on E17.5 and processed for anti-BrdU immunohistochemistry.

Purification of Sema4D and semaphorin binding assay. Recombinant semaphorins were produced by transient transfection in COS cells, and enriched preparations were obtained from cell-conditioned media, as described previously (Tamagnone et al., 1999; Barberis et al., 2004).

In situ binding assays were performed as described previously (Tamagnone et al., 1999). Briefly, COS cells were transiently transfected to express plexin-B1, plexin-B2 (HA-tagged), plexin-B3 (VSV-tagged), or green fluorescent protein (GFP), as mock control. Forty-eight hours after transfection, cells were incubated for $1 \mathrm{~h}$ at $37^{\circ} \mathrm{C}$ with the recombinant secreted semaphorins fused to AP (Sema-AP). After extensive washes, cells were fixed, heated for $10 \mathrm{~min}$ at $65^{\circ} \mathrm{C}$ to inactivate endogenous phosphatases, and incubated with NBT-BCIP (nitro blue tetrazolium-5bromo-4-chloro-3-indolylphosphatase) AP substrate (Promega, Madison, WI). Binding kinetics were determined by incubating plexinexpressing cells with increasing concentrations of the ligands and measuring cell-bound semaphorins by means of AP substrate 4-nitrophenyl phosphate (Sigma catalog \#S0942), as described previously (Flanagan and Leder, 1990; Artigiani et al., 2003). Scatchard plot analysis was performed using Equilibrate. The expression of different plexins in COS cells used for binding and collapse assays (below) was verified by Western blotting on equal amounts of total protein lysates.

COS cell collapse assay. COS cells transiently transfected to express different plexins (as above) were grown to subconfluency overnight on poly-L-lysine-coated coverslips and then incubated for $1 \mathrm{~h}$ at $37^{\circ} \mathrm{C}$ with recombinant-secreted semaphorins (or control media). Cell monolayers were then fixed with $4 \%$ PFA, permeabilized with $0.2 \%$ Triton X-100, blocked with $2 \%$ goat serum, and eventually incubated with specific antibodies to detect plexin-expressing cells (EC6.9 for plexin-B1 and anti-HA for plexin-B2). Signal detection was done with Alexa Fluorconjugated anti-mouse antibodies. The number of plexin-expressing collapsed COS cells (defined as immunostaining-positive rounded cells with the major diameter of $\leq 30 \mu \mathrm{m}$ ) was counted independently by two investigators, in two separate experiments, including duplicate wells for each condition ( $n>100$ /experiment).

Generation of a plasmid expressing dominant-negative plexin-B2. Amino acids 1-1220 of plexin-B2, containing the extracellular domain and transmembrane region were fused to Myc tag and cloned in pcDNA3 vector. 
Determination of activate RhoA. The amount of activated RhoA was determined by precipitation of a fusion protein consisting of GST and the Rho binding domain of Rhotekin (GSTRBD), as described originally by Ren and Schwartz (Ren and Schwartz, 2000). Fortyeight hours after transfection, cells were starved for $12 \mathrm{~h}$, and then treated with PBS, purified Sema4D, or Sema4C-AP medium. After 20 min, cells were lysed and GTP-bound Rho was precipitated as described previously in details (Swiercz et al., 2002). RhoA was detected by immunoblotting using a monoclonal antiRhoA antibody.

Data analysis and statistics. All data are represented as mean \pm SEM. Data were statistically analyzed by performing an ANOVA using random measures followed by Fisher's post hoc test.

\section{Results}

Plexin-B1 and Plexin-B2 are expressed in premigratory and migrating granule cells

We found that a hallmark feature of plexin-B2 mRNA is its abundance in GCPs of the developing cerebellum, dentate gyrus, and olfactory bulb. During the first postnatal week, plexin-B1 continues to be expressed after granule cells migrate from the EGL (Fig. $1 A$, arrowheads) into the internal granular layer (IGL) (arrows), whereas the expression of plexin-B2 ceases in cells that have completed migration into IGL. We therefore analyzed the time course of plexin-B2 expression in more detail and found that both GCPs in the outer, proliferative sublayer of the EGL (Fig. $1 B$, dashed lines) as well as postmitotic cells in the premigratory sublayer of the EGL (identified via TAG-1 immunoreactivity) express plexin-B2 mRNA (Fig. $1 B)$. In the developing cerebellum, Sema4d mRNA is expressed in migrating oligodendrocytes, not in the vicinity of GCPs (Worzfeld et al., 2004). GCP migration is also prominent in the developing dentate gyrus of the hippocampus over E13-E17.5. In contrast to plexin-B1, which is expressed in the neuroepithelium and the pyramidal cell layer of the hippocampus, plexin-B2 mRNA is strongly expressed in a layer of cells that lies between the lateral ventricle and the pyramidal cell layer (Worzfeld et al., 2004) (Fig. 1C). Immunostaining for NeuroD, a neurochemical marker for migrating GCPs, and in situ hybridization for plexin-B2 on serial sections derived from E17.5 brains revealed that this layer of plexin-B2-expressing cells represents the migratory stream of GCPs into the dentate gyrus (Fig. $1 D)$. As with the cerebellum, it is striking that the expression of plexin-B2 ceases sharply in GCPs that have reached their target zone in the dentate gyrus and differentiate (Fig. $1 D$ ). Interestingly, Sema4d expression was complementary to and in the close proximity of GCPs in the proximal dentate migratory stream (Fig. 1C). Via the rostral migratory stream (RMS), GCPs also migrate from the lateral ventricle into the developing olfactory bulb. We observed that plexin-B1 and plexin-B2 are expressed in the RMS at embryonic and early postnatal stages (Fig. 1E). As with the cerebellum, Sema4d expression was not detectable in the vicinity of the GCPs in the RMS (data not shown).

Effects of Sema4D on adhesion, proliferation, and migration of granule cell precursors

To elucidate the functional significance of Plexin-B1 and Plexin-B2 proteins in GCPs, we applied preparations of the soluble, N-terminal domain of Sema4D protein to GCPs isolated from cerebellar EGL on P5. Sema4D was used either as a purified preparation ( $\mathrm{pSema4D)}$ after column purification of His-tagged Sema4D (Barberis et al., 2004) or as medium concentrated from HEK293 cells secreting soluble, Sema4D-AP (Swiercz et al., 2004). Medium concentrated from vector-transfected HEK293 

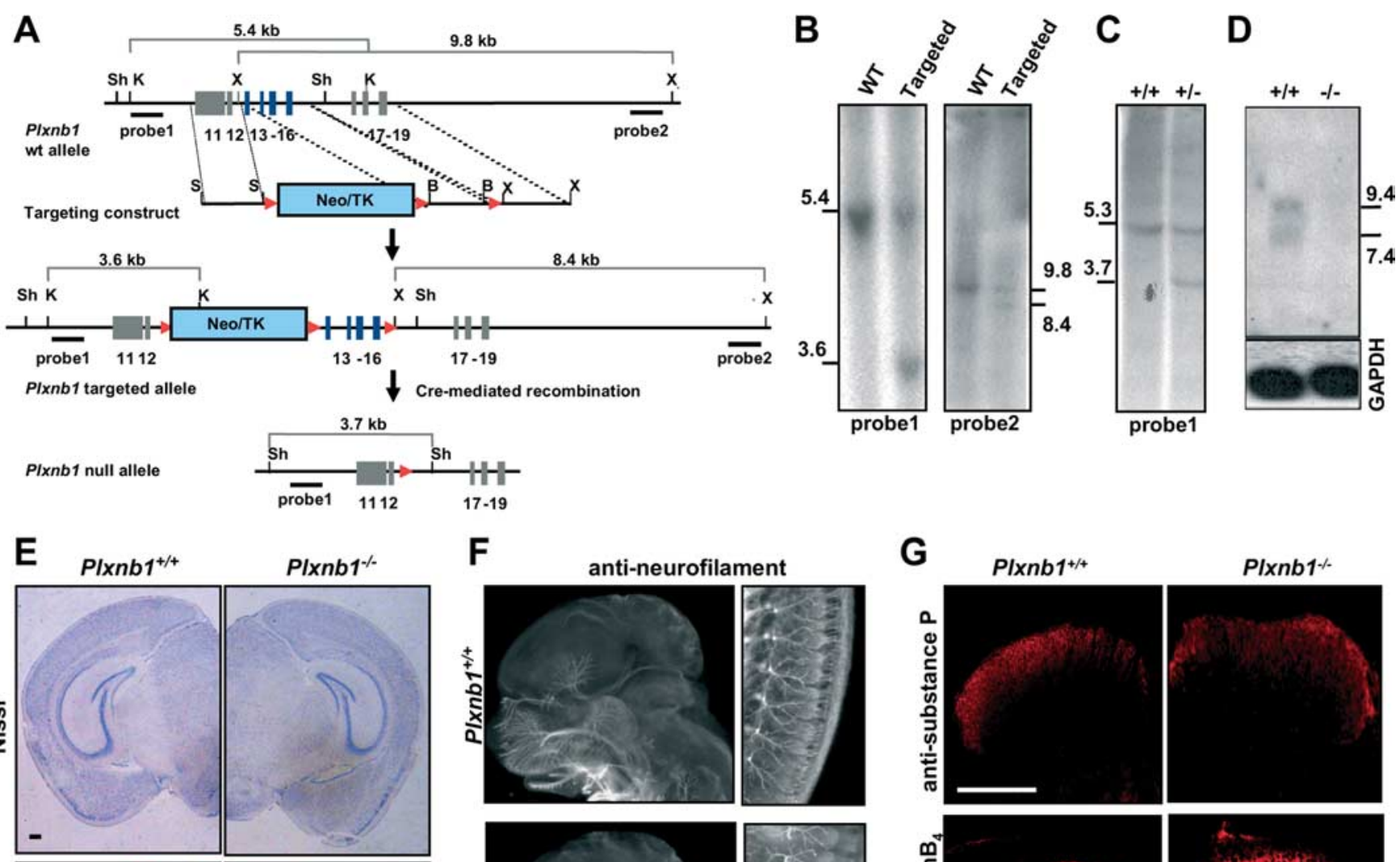

$\mathbf{F}$

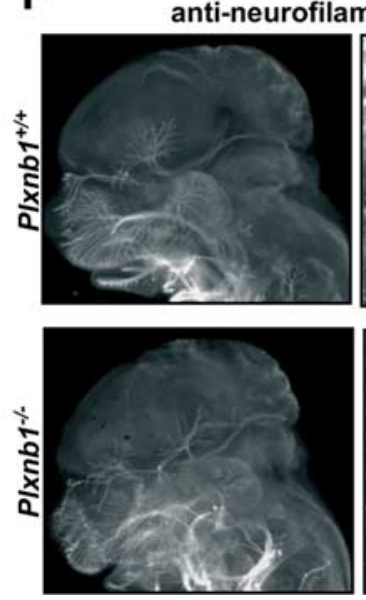

G

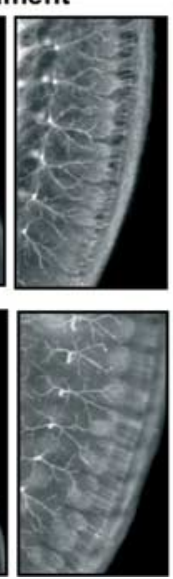

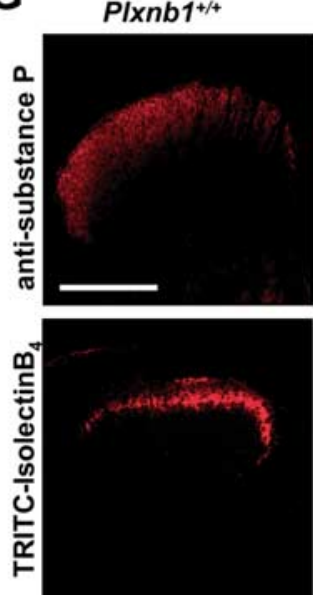

Plxnb1\%

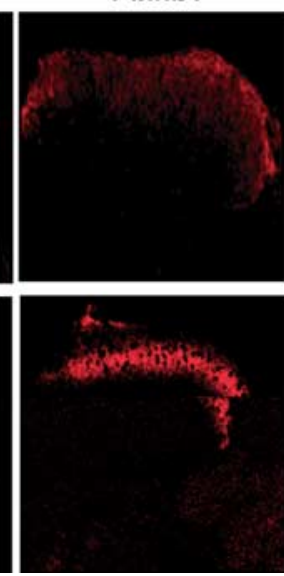

Figure 2. Generation and characterization of mice lacking Plexin-B1 in a constitutive manner ( $\left.p / x n b 1^{-1-}\right) \cdot A$, Targeting strategy for generation of mice lacking exons $13-16$ of the $p / x n b 1$ gene. Red triangles indicate loxP sites. K, Kpnl; Sh, Sph; X,Xhol. B, Southern blot analysis of correctly targeted ES cell clones analyzed according to the scheme shown in $\boldsymbol{A}$. WT, Wild type. C, Southern blot analysis of heterozygous mutant mice and wild-type littermates analyzed according to the scheme shown in $\boldsymbol{A}$. $\boldsymbol{D}$, Demonstration of the loss of plexin-B1 mRNA transcript in the brain of adult homozygous mice at E17.5 via Northern blot analysis. The numbers indicate size in kilobase in $\boldsymbol{B}-\boldsymbol{D}$. E, Nissl staining and anti-CNPase immunohistochemistry show that the gross morphology of the brain nuclei and the fiber tracts, respectively, are unaltered in adult $p l x n b 1^{-1-}$ embryos compared with wild-type littermates. $\boldsymbol{F}$, Whole-mount anti-neurofilament $M$ immunostaining reveals normal trajectories of cranial (left panels) and spinal nerves (right panels) plxnb1 ${ }^{-1-}$ embryos and wild-type littermates at E12. G, Spinal connectivity of populations of sensory nerves identified via anti-substance $P$ immunoreactivity and Isolectin-B4 binding is developed normally in plxnb1 ${ }^{-1-}$ mice. Scale bars, $25 \mu \mathrm{m}$.

cells served as a control (mock). Neither pSema4D nor Sema4D-AP had any significant effects on adhesion of acutely dissociated GCPs to the culture dishes coated with poly-L-lysine, laminin, or fibronectin substrates (Fig. $1 F)(p>0.05)$. Furthermore, Sema4D failed to influence proliferation of GCPs, as judged via BrdU incorporation (Fig. $1 G)(p>0.05)$. In contrast, Sema4D stimulated migration of dissociated GCPs to the same extent as SDF- $1 \alpha$, a potent promigratory cue for GCPs, as seen in Boyden chamber assays (Fig. $1 H)(p<0.001$ compared with control medium). Similar results were obtained with SVZ explants cultured from the lateral wall of the anterior horn of the lateral ventricle, the origin of the RMS. Sema4D-AP stimulated chain migration of RMS neuroblasts (immunoreactive for PSANCAM) from SVZ explants, as assessed by measuring area of migration $\left(1.18 \pm 0.11 \mu \mathrm{m}^{2}\right.$ in Sema4D-treated group versus $0.602 \pm 0.10 \mu \mathrm{m}^{2}$ in the mock-treated group; $\left.p<0.001\right)$.

\section{Generation of Plexin-B1 knock-out mice}

To generate mice lacking Plexin-B1, the exons 13-16 of the murine plxnb1 gene were deleted (for details, see Materials and
Methods and Fig. 2A-C). The Plexin-B1 mRNA transcript could not be detected via Northern blot analysis in the brain of the homozygous progeny ( plxnb1 $1^{-1-}$ mice) (Fig. $2 D$ ), which was further confirmed by mRNA in situ hybridization (supplemental Fig. 1, available at www.jneurosci.org as supplemental material). The expression levels of Plexin-B2 mRNA transcripts did not change in the brains of plxnb1 $1^{-1-}$ mice (data not shown). $P l x n b 1^{-1-}$ mice were found to be viable and fertile and did not show any obvious phenotype or defects in the organization of major cellular groups or fiber tracts in the brain (Fig. $2 E$ ). Furthermore, whole-mount immunostaining of E12 embryos for neurofilament-M did not reveal any deviations from the expected peripheral innervation patterns of cranial and spinal nerves in plxnb1 $1^{-1-}$ mice (Fig. $2 F$ ). Similarly, the terminals of peptidergic and nonpeptidergic small-diameter sensory neurons, judged by anti-substance $\mathrm{P}$ immunoreactivity and binding to TRITClabeled Isolectin-B4, respectively, were found in their proper targets in the superficial spinal dorsal horns of adult $p l x n b 1^{-1-}$ mice (Fig. 2G). 


\section{Plexin-B1 is not essential for cerebellar and olfactory bulb development}

In-depth analyses of cerebellar and olfactory bulb development using several in vitro and in vivo assays for GCP migration and proliferation failed to reveal any developmental defects in plxnb1 $1^{-1-}$ mice.

We analyzed the migration of GCPs and the development of cerebellar lamination in $p l x n b 1^{-1-}$ mice. There was no difference between $p l x n b 1^{+1-}$ and $p l x n b 1^{-1-}$ mice with respect to the ability of Sema4D to stimulate the migration of dissociated GCPs from the EGL in Boyden chamber assays at P5 (supplemental Fig. $2 A$, available at www.jneurosci.org as supplemental material) (SDF-1 $\alpha$ serves as a positive control). However, we found that Sema4D stimulated migration of granule cells out of explants derived acutely from the EGL in plxnb1 $1^{+/-}$mice but not in plxnb1 $1^{-1-}$ mice at P5 (supplemental Fig. $2 B$, available at www. jneurosci.org as supplemental material). Thus, plexin-B1 was found to be involved in the migration of GCPs out of the EGL explants but was not required for the stimulatory effects of Sema4D on dissociated GCPs, suggesting that plexin-B1 may only indirectly modulate some aspects of GCP migration. To study this process in a more physiological context, we analyzed migration of GCPs in organotypic slice cultures derived from the

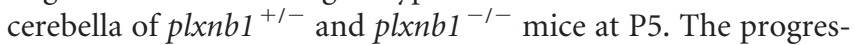
sive depletion of the anti-NeuroD-immunoreactive EGL, as granule cells migrated into the IGL, occurred to a similar extent in slices derived from $p l x n b 1^{+/-}$mice and $p l x n b 1^{-1-}$ mice (supplemental Fig. $2 C$, available at www.jneurosci.org as supplemental material). Furthermore, in vivo BrdU-labeling studies revealed that both proliferation and migration of cerebellar GCPs, assessed at 1 and $2 \mathrm{~h}$ after BrdU injection, respectively, were comparable across $p l x n b 1^{+/-}$and plxnb1 $1^{-1-}$ mice at P5 (supplemental Fig. 2D, available at www.jneurosci.org as supplemental material). Finally, normal cerebellar development in plxnb1 ${ }^{-1-}$ mice at P5 or P11 in vivo was observed using a variety of cell- or layer-specific markers, such as NeuroD (labels EGL), NeuN (labels newly differentiated granule cells in the IGL), calbindin (labels Purkinje cells), and GFAP (labels astrocytes) (supplemental Fig. 2E, available at www.jneurosci.org as supplemental material). These results demonstrate that plexin-B1 is not strictly required for cerebellar development.

Migration in the RMS and the development of the olfactory bulb is unaltered in plxnb1 $1^{-1-}$ mice. In explant cultures derived from the SVZ of the RMS at P5, we observed that Sema4D treatment stimulated chain migration of neuronal precursors to a

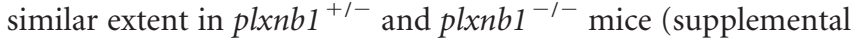
Fig. $3 A$, available at www.jneurosci.org as supplemental material). Furthermore, BrdU-labeling experiments failed to demonstrate any differences between $p l x n b 1^{+/-}$and $p l x n b 1^{-1-}$ mice with respect to the proliferation and migration of neuronal precursors in the RMS at P5 in vivo (supplemental Fig. $3 B$, available at www.jneurosci.org as supplemental material). The structure of the olfactory bulb was entirely normal in $p l x n b 1^{-1-}$ mice (supplemental Fig. 3C, available at www.jneurosci.org as supplemental material). Moreover, the distribution and patterning of various types of neurons in the olfactory bulb was similar in plxnb1 $1^{+1-}$ and plxnb1 $1^{-1-}$ mice, as assessed via immunoreactivity for tyrosine hydroxylase, which labels the periglomerular cell layer, GAD67, which labels the granule cell and periglomerular cell layers as well as calbindin and calretinin, which label populations of interneurons (supplemental Fig. 3C, available at www. jneurosci.org as supplemental material) (data not shown). Furthermore, the projection of the olfactory nerves to the olfactory bulb was normal, as judged by GAP-43 expression [supplemental Fig. 3C (arrow), available at www.jneurosci.org as supplemental material]. These results demonstrate that plexin-B1 deficiency does not influence RMS migration and olfactory bulb development.

\section{A loss of Plexin-B2 leads to defects in neural tube closure and to exencephaly}

Because our extensive analysis did not reveal a requirement for Plexin-B1 in development and Sema4D-induced effects on GCP migration, we generated mice lacking Plexin-B2 (see Materials and Methods and Fig. 3A-D), the only other plexin-B family member that is highly expressed in neuronal progenitors (Worzfeld et al., 2004). Plxnb2 $2^{-1-}$ mice entirely failed to express plexin-B2 mRNA as observed via Northern blot analysis (Fig. 3D) and in situ hybridization (supplemental Fig. 1, available at www.jneurosci.org as supplemental material).

In striking contrast to $p l x n b 1^{-1-}$ mice, $p l x n b 2^{-1-}$ mice demonstrated severe developmental abnormalities. Mating of plxnb2 $2^{+/-}$mice did not yield any viable homozygous knock-out offspring. Analysis of embryos showed that 41 of 207 embryos were plxnb2 $2^{-1-}(19.8 \%)$. Of these, 36 showed developed neural tube defects (exencephaly, see below), and five showed a normal topology of the brain and spinal cord. Thus, the penetrance of the exencephaly phenotype was calculated to be $87.8 \%$. Examination at E9.5 revealed that the cranial neural folds did not converge at the midline, resulting in an open neural tube (Fig. $3 E$, arrows) $(n=5)$, which was confirmed in Nissl-stained sections of the E9.5 embryos (Fig. $3 F$ ). Other neural tube-associated defects such as spina bifida were observed in only 2 of 41 plxnb2 $2^{-1-}$ embryos, and microscopic analysis revealed a normal closure of the neural tube at the spinal cord level in all other cases (Fig. $3 F$ ). At E13.5 (data not shown) and E17.5 (Fig. 3E), there was a prominent protrusion of the brain in the absence of the cranial vaults, resulting in the "open-brain" phenotype or exencephaly (Fig. 3E, arrowheads) $(n=31)$. Histological analysis revealed that exencephalic plxnb2 $2^{-1-}$ mutants displayed an inversed topography of the brain consisting of a cortex at the base of the cranium with overlying prominent diencephalic expansions (Fig. $3 G)(n=5)$. The organization of the striatum and the olfactory bulb (arrow) was entirely disrupted (Fig. 3G). There was a conspicuous enlargement of the ventricles and a thinning of the proliferative ventricular zone (Fig. $3 G$, arrowheads) $(n=5)$ accompanied by multiple ventricular defects. We then took a closer look at the ventricular defects, which were prevalent in plxnb2 $2^{-1-}$ embryos that displayed exencephaly as well as those that did not. The ventricular wall was discontinuous at several places accompanied by large protrusions of the neuronal parenchyma into the ventricles [supplemental Fig. $4 \mathrm{~A}$ (arrowheads), available at www. jneurosci.org as supplemental material]. Furthermore, ventricular ectopias were found in the lateral ventricles of $p l x n b 2^{-1-}$ mice in the form of large aggregates consisting of viable cells (DAPIpositive) as well as cellular debris (supplemental Fig. $4 \mathrm{~A}$, available at www.jneurosci.org as supplemental material). Surprisingly, the viable cells in ventricular ectopias were immunoreactive for a variety of proteins that label different populations of neuroblasts as well as differentiated cells, such as PSANCAM, neuron-specific $\beta$-tubulin III (data not shown), GAD67 , and GFAP [supplemental Fig. $4 B$ (arrowheads), available at www.jneurosci.org as supplemental material]. Furthermore, plxnb2 $2^{-1-}$ embryos with exencephaly demonstrated a prominent hypertrophy of the choroid plexus in the lateral ventricles [supplemental Fig. $4 A$ (asterisk), available at www.jneurosci.org as 
A

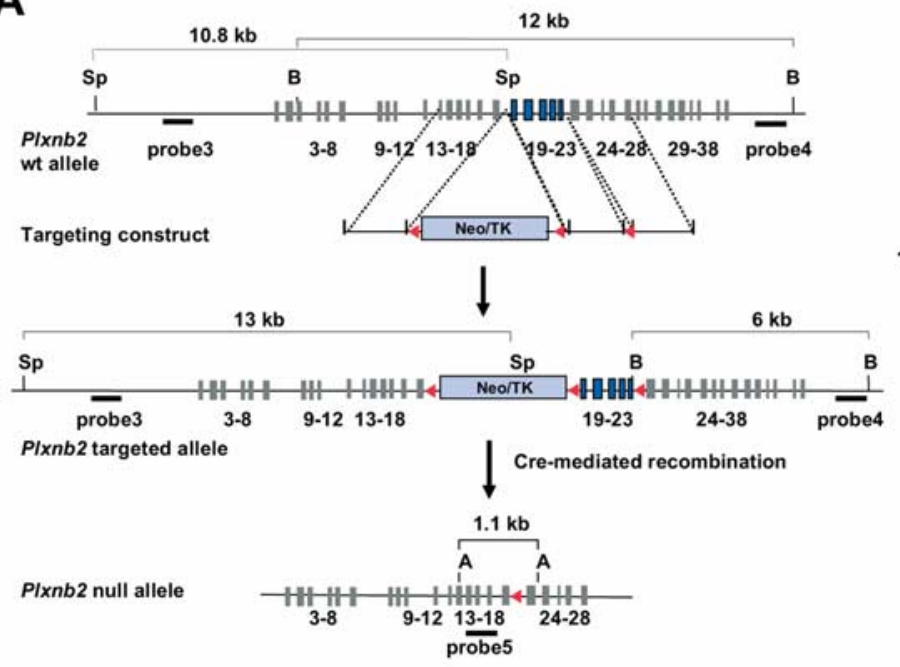

B
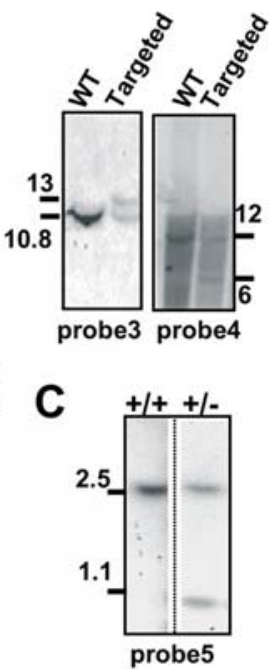

D

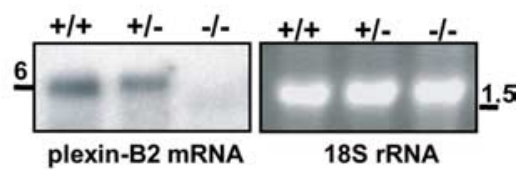

E

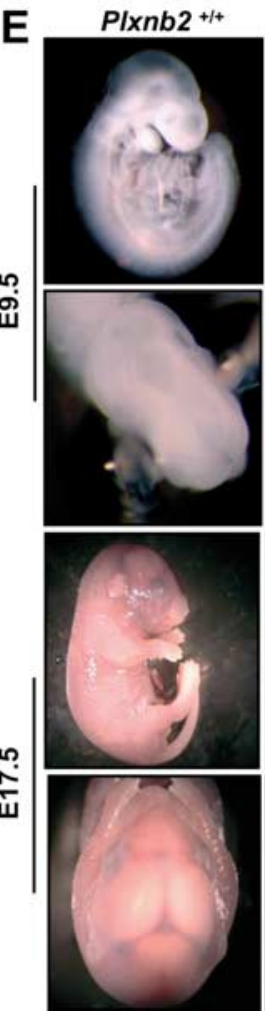

PIxnb2 \%
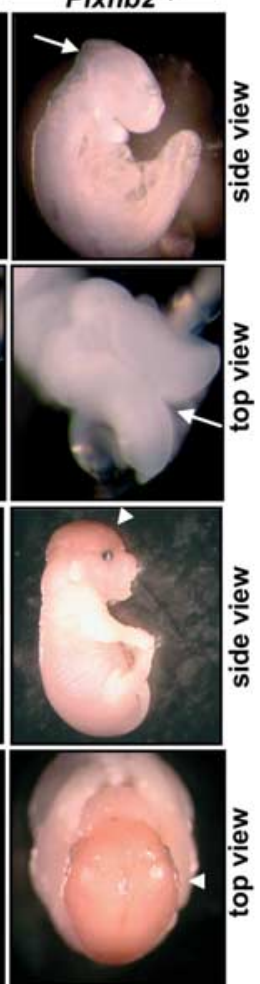

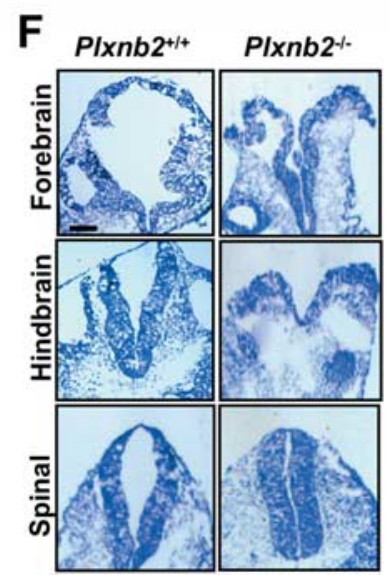

G

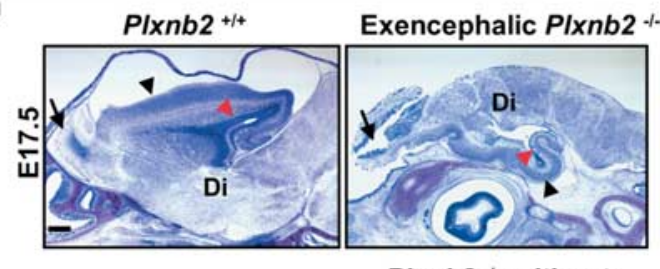

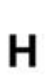

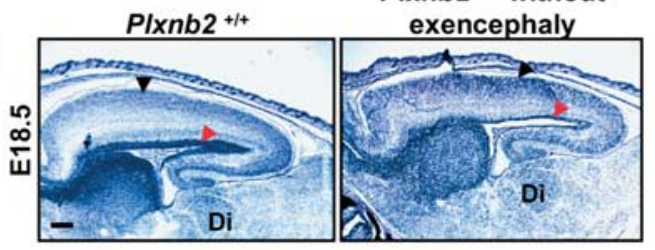

Figure 3. Generation and characterization of mice lacking Plexin-B2 in a constitutive manner. $A$, Targeting strategy for generation of mice lacking exons $19-23$ of the $p / x n b 2$ gene. Red triangles indicate loxP sites. Sp, Spel; B, BamHI; A, Asel. B, Southern blot analysis of correctly targeted ES cell clones analyzed according to the scheme shown in $A$. WT, Wild type. C, Southern blot analysis of heterozygous mutant mice and wild-type littermates analyzed according to the scheme shown in $A$. D, Demonstration of the loss of plexin-B2 mRNA transcript in the brain of homozygous embryos at E17.5 via Northern blot analysis. The numbers indicate size in kilobase in $\boldsymbol{B}-\boldsymbol{D}$. $\boldsymbol{E}$, Gross morphology of $p / x n b 2^{-/-}$embryos and wild-type littermates at E9.5. Plxnb2 ${ }^{-/-}$embryos demonstrate an open neural tube (arrows) and exencephaly (arrowheads). $\boldsymbol{F}$, Nissl staining on coronal sections of plxnb2 ${ }^{-1-}$ embryos and wild-type littermates at E9.5 shows an open neural tube in the forebrain and hindbrain regions, but not in the spinal neural tube, in plxnb2 ${ }^{-1-}$ embryos $(n=5)$. G, Nissl staining on sagittal sections of $p / x n b 2^{-1-}$ embryos and wild-type littermates at E17.5 reveals an inversion of the brain (black arrowheads) with an overlying diencephalon (Di), enlarged ventricles, and hypotrophic ventricular germinative zone (red arrowheads) and a disruption of the rostral migratory stream and olfactory bulb (black arrows) in plxnb2 ${ }^{-1-}$ embryos $(n=5) . \boldsymbol{H}$, Some plxnb2 ${ }^{-1-}$ embryos do not develop exencephaly (black arrowheads) but still show enlarged ventricles and hypotrophic ventricular germinative zone (red arrowheads; $n=4$ ). Scale bars, $25 \mu \mathrm{m}$.

supplemental material]. Interestingly, enlargement of the ventricles and the hypotrophy of the ventricular zone (Fig. $3 H$, arrowheads) were also evident in plxnb2 $2^{-1-}$ embryos, which did not develop exencephaly and showed a normal topology of the brain (Fig. $3 H)(n=4)$.

\section{Abnormal development of the dentate gyrus in plxnb2 $2^{-/-}$mice}

Because Plexin-B2 mRNA is prominently expressed in the migratory stream of GCPs in the developing hippocampus (Fig. $1 E$ ), we studied the development of the dentate gyrus in $p l x n b 2^{-1-}$ mice. Compared with plxnb2 $2^{+/+}$embryos, plxnb2 $2^{-1-}$ embryos at E17.5 demonstrated a severe reduction in the number of GCPs in the stream migrating toward the developing dentate gyrus (Fig. $4 A$, arrows) $(n=4)$ in Nissl-stained sections. Furthermore, whereas neat rows of radially migrating neuroblasts originating from the germinative ventricular zone facing the Ammon's horn were evident in plxnb2 $2^{+/+}$embryos, these migratory patterns were entirely disbanded in mutant embryos (Fig. $4 A$, bottom, arrowheads), accompanied by disruption of the ventricular wall (Fig. $4 A$, bottom, yellow arrow) $(n=4)$.

In plxnb2 $2^{+/+}$embryos at E17.5, migrating GCPs identified via NeuroD staining had either reached their destination in the developing dentate gyrus anlage or were found in the subpial migratory route (Fig. $4 \mathrm{~B}$, top left, arrow). In contrast, large numbers of NeuroD-immunoreactive GCPs were found in proximal parts of the migratory stream (arrowheads) in plxnb2 $2^{-1-}$ embryos with exencephaly (Fig. $4 B$, top) $(n=3)$. A similar retardation of GCP migration was observed at E18.5 in plxnb2 ${ }^{-1-}$ embryos that did not develop exencephaly (Fig. $4 B$, bottom) $(n=3)$. In contrast, age-matched plxnb2 $2^{+/+}$littermates only retained a few NeuroD-positive granule cells in the dentate gyrus anlage (Fig. $4 B$, bottom left, dashed arrow) $(n=3)$, consistent with the maturation of the dentate gyrus. Furthermore, prox-1-expressing differentiating granule cells were found in reduced numbers in the dentate gyrus of plxnb2 $2^{-1-}$ embryos compared with plxnb2 $2^{+1+}$ embryos at E17.5 (arrowheads) and were detected ectopically in distal regions of the stratum lacunosum moleculare 
(arrows) (Fig. 4C, top right). Furthermore, compared with wild-type littermates, neuron-specific $\beta$-tubulin IIIexpressing neuroblasts were patterned abnormally in plxnb2 $2^{-1-}$ embryos (Fig. $4 C$, bottom right, arrowheads) $(n=3)$ and formed ectopic aggregates (Fig. 4C, arrows, magnified in inset). GFAPexpressing radial glial cells, which guide the migration of GCPs during embryonic development (Yuasa, 2001), were evident at appropriate locations in subpial region (data not shown) and the ventricular zone of the developing hippocampus in plxnb2 $2^{-1-}$ embryos, albeit their morphology was abnormal (Fig. 4D). Together, these results demonstrate that Plexin-B2 is critically required for GCP migration and dentate gyrus development.

\section{Defects in cerebellar foliation and lamination in plxnb2 $2^{-/-}$mice}

We then addressed the involvement of Plexin-B2 in early, embryonic processes of cerebellar development (Fig. 5). Unlike plxnb2 $2^{+/+}$embryos, which demonstrated a clear primordial cerebellum consisting of an EGL (arrowheads) and a developing Purkinje cell layer (Fig. 5A, arrow) at E17.5, plxnb2 $2^{-1-}$ embryos only showed a partially formed cerebellar primordium with an incomplete EGL (arrowhead) in Nissl-stained sections (Fig. $5 A)(n=5)$. Consistent with these observations, EGL cells at embryonic stages E15.5 and E17.5 were found to strongly express plexin-B2 mRNA (Fig. 5B). Anti-NeuroD immunohistochemistry confirmed that in contrast to plxnb2 $2^{+1+}$ embryos, plxnb2 $2^{-1-}$ embryos showed on-going GCP migration from the rhombic lip (Fig. 5C, arrows) into the nascent EGL (Fig. 5C, arrowheads) $(n=3)$. Moreover, plxnb2 $2^{-1-}$ embryos demonstrated striking deficits in the foliation of the developing cerebellum. Whereas plxnb2 $2^{+/+}$embryos had developed cerebellar foliation with four fissures by E18.5, plxnb2 $2^{-1-}$ embryos did not show any folia $(n=5)$. The EGL of plxnb2 $2^{-1-}$ embryos remained thin at E18.5, and NeuroD-labeled GCPs demonstrated a patchy and irregular distribution in the EGL (Fig. $5 C$, bottom right, arrowheads) $(n=3)$, in contrast to plxnb2 $2^{+/+}$cerebellum, in which migration of GCPs into the IGL from a correctly patterned EGL had already commenced (Fig. 5C, bottom left, arrowheads). Finally, TAG-1 immunostaining revealed that the layer of premigratory granule cells was well formed in the inner aspect of the EGL of plxnb2 $2^{+/+}$embryos but was not evident in plxnb2 $2^{-1-}$ embryos. (Fig. 5D). Importantly, cerebellar defects were found in plxnb2 $2^{-1-}$ embryos exhibiting exencephaly as well as in those that did not develop exencephaly $(n=3)$.
A

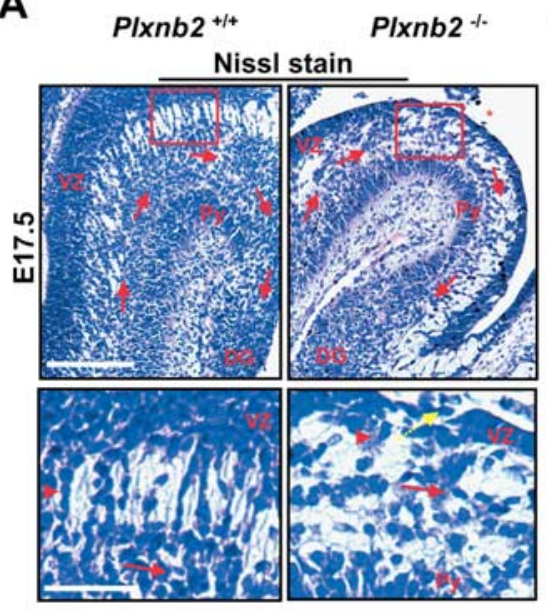

B Plxnb2 ${ }^{+/+} \quad$ Plxnb2 $\%$

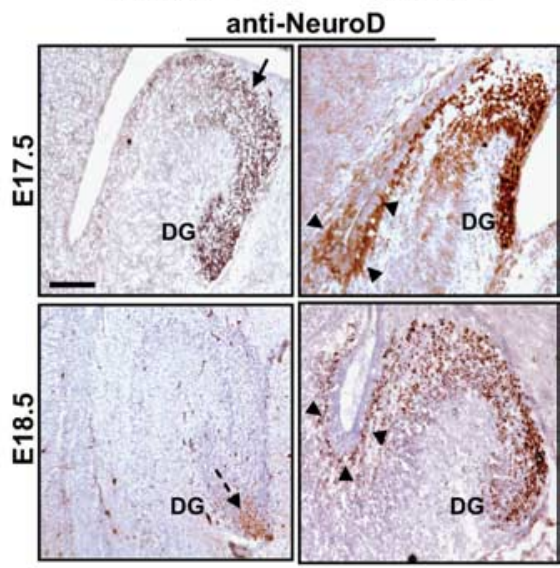

C

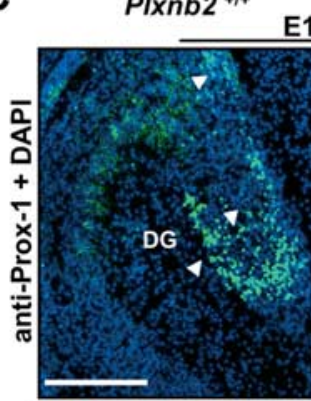

E17.5 PIxnb2\%

D
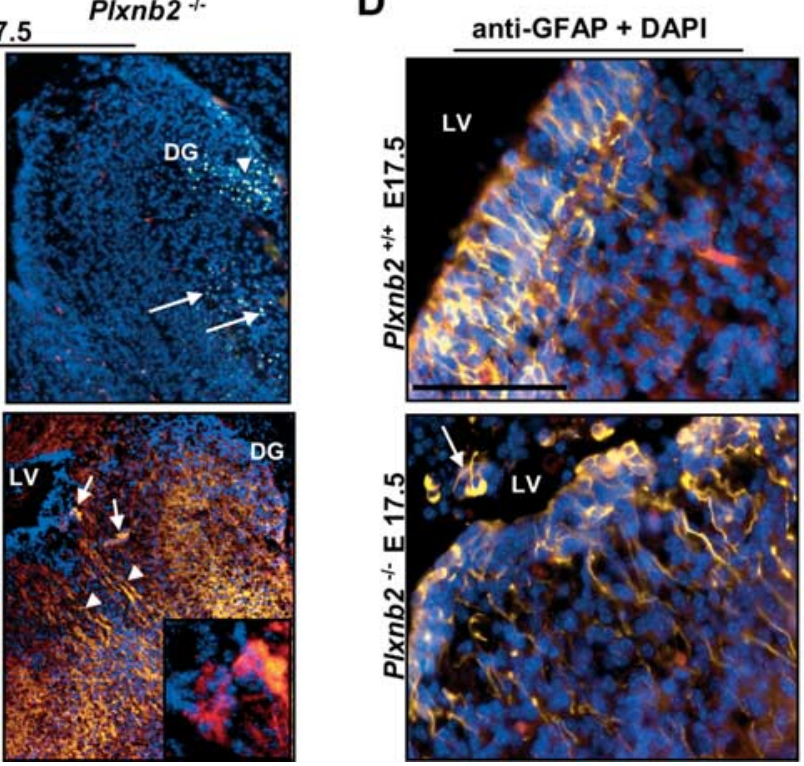

Figure 4. Characterization of the defects in development of the dentate gyrus in $p / x n b 2^{-1-}$ embryos at E17.5 and E18.5.A, The migratory stream of GCPs (marked by red arrows) is severely reduced and disoriented in plxnb2 ${ }^{-1-}$ embryos. Boxed areas at the top are magnified in the lower panels. Py, Developing pyramidal cell layer; DG, dentate gyrus. Red arrowheads indicate radially migrating neuroblasts: yellow arrow points to the disrupted ventricular wall in $p / x n b 2^{-I-}$ embryos. $\boldsymbol{B}$, Retarded migration (black arrowheads) of NeuroD-expressing GCPs into the DG anlage. NeuroD immunoreactivity is decreased in wild-type littermates at E18.5 and restricted to the DG (dashed arrow). The E18.5 plxnb2 $2^{-1-}$ embryo shown here did not develop exencephaly. $C$, Differentiating granule cells identified via anti-Prox-1 immunoreactivity (green) in the developing DG (white arrowheads) are reduced in plxnb2 $2^{-1-}$ embryos and found in ectopic locations (white arrows). Immunoreactivity toward neuron-specific $\beta$-tubulin III (orange) reveals a disruption of the migratory pattern of neuroblasts (white arrowheads) in plxnb2 $2^{-1-}$ embryos accompanied by ectopic $\beta$-tubulin III-expressing cellular aggregates (white arrows, magnified in inset). $\boldsymbol{D}$, GFAP-expressing radial glia are present in plxnb2 ${ }^{-1-}$ embryos but demonstrate abnormal morphology and a disruption of the ventricular wall with ectopically located cells in the lateral ventricle (LV). Scale bars: $\boldsymbol{D}, 10 \mu \mathrm{m} ; \boldsymbol{B}-\boldsymbol{D}, 25 \mu \mathrm{m} . n=3 \mathrm{plxnb2^{-1- }}$ embryos each with or without exencephaly and equal number of wild-type controls.

\section{Development of the olfactory bulb is retarded in} plxnb2 $2^{-1-}$ mice

Because the olfactory bulb was severely distorted in exencephalic plxnb2 $2^{-1-}$ mice, we used plxnb2 $2^{-1-}$ mice that did not develop exencephaly to analyze the contribution of Plexin-B2 to cell migration along the RMS and olfactory bulb development. Nuclear DAPI staining revealed a retardation of lamination in the olfac-

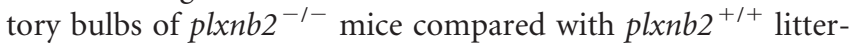
mates at E18.5 (Fig. 6A, top panel) $(n=3)$. Furthermore, whereas PSA-NCAM-expressing neuronal progenitors were evi- 

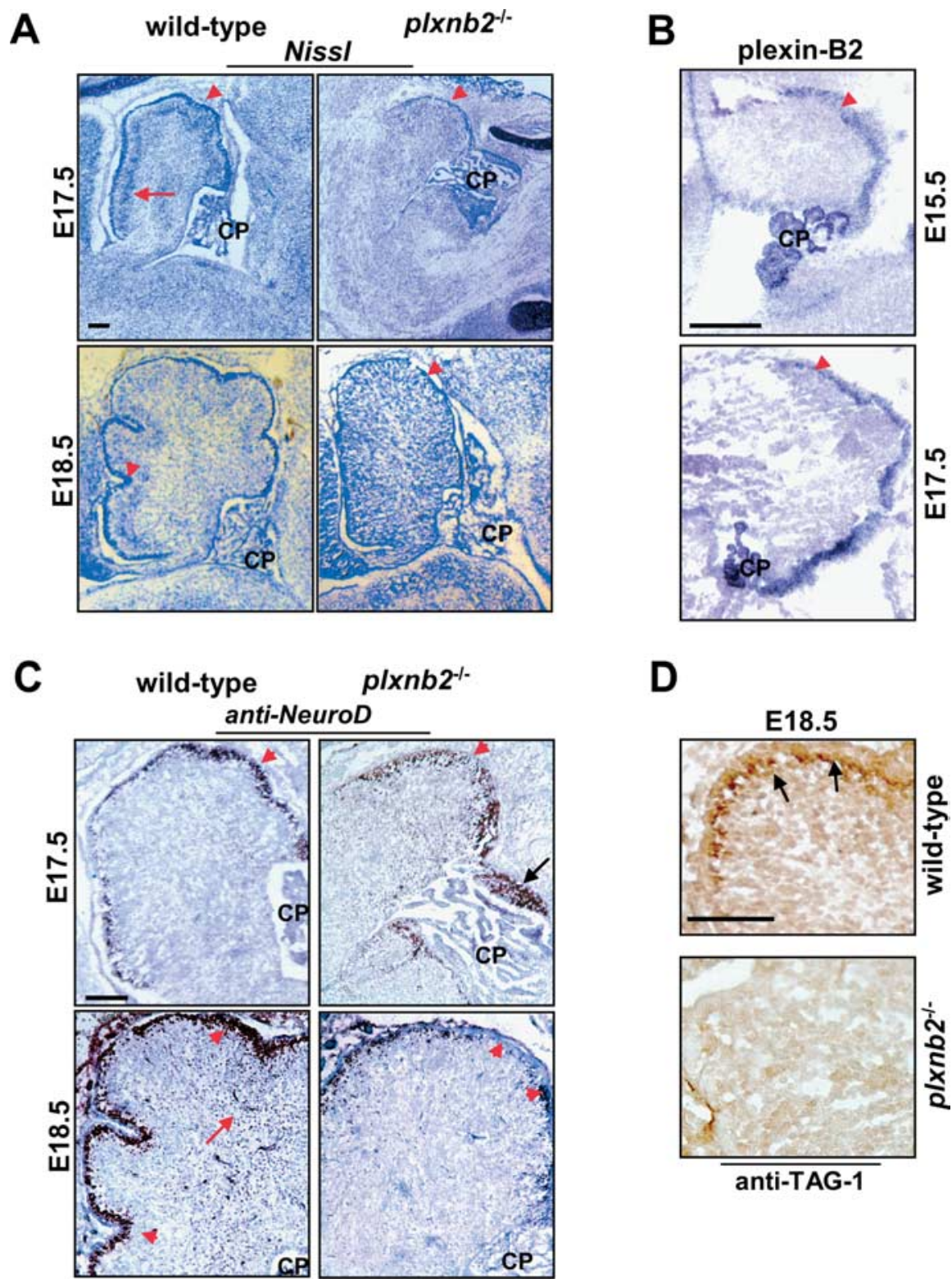

Figure 5. Analysis of defects in GCP migration and cerebellar foliation in plxnb2 ${ }^{-/-}$mice at E17.5 and E18.5. A, Nissl staining revealed a retarded development of the cerebellar primordium and EGL in $p l x n b 2^{-1-}$ mice compared with wild-type ( $p / x n b 2^{+/+}$) littermates, which showed a clear EGL (arrowheads, top panel) and a developing layer of Purkinje cells (arrow). Cerebellar foliation (arrowheads) is clearly evident in wild-type mice at E18.5 but not in $p / x n b 2^{-1-}$ mice, including those that did not develop exencephaly. $\boldsymbol{B}$, plexin-B2 mRNA is detected via in situ hybridization in the cerebellar anlage, EGL, and choroid plexus (CP) at E15.5 and E17.5. C, At E17.5, NeuroD-expressing GCPs are clearly patterned in the EGL of wild-type mice, whereas plxnb2 $2^{-1-}$ mutants demonstrate an irregular EGL (arrowheads) and cells that had not migrated into the EGL (black arrow). Migration of granule cells into the IGL is evident in wild-type mice at E18.5 (red arrow) but not in plxnb2 ${ }^{-1-}$ embryos. D, TAG-1 immunostaining reveals a layer of differentiating premigratory granule cells (arrows) in wild-type mice but not in plxnb2 $2^{-1-}$ mice. Scale bars, $25 \mu \mathrm{m} . n=3 \mathrm{p} / \mathrm{xnb}^{-1-}$ embryos each with or without exencephaly and equal number of wild-type controls.

dent in the developing laminas of the olfactory bulb of plxnb2 $2^{+/+}$ embryos, plxnb2 $2^{-1-}$ embryos only demonstrated PSA-NCAMexpressing cells in the RMS (Fig. $6 A$, middle) $(n=3)$. A similar retardation of migration was observed in plxnb2 $2^{-1-}$ embryos with respect to GAD-67-expressing cells in the olfactory bulb (Fig. $6 A$, bottom $)(n=3)$.

\section{Impaired neuronal proliferation in $p l x n b 2^{-1-}$ mice}

The striking hypotrophy of the ventricular zone and the EGL, both of which represent zones of high proliferative activity, in plxnb2 $2^{-1-}$ embryos prompted us to address whether prolifera- tion of neuroblasts is affected by Plexin-B2 deficiency. Immunostaining for Ki-67, a cell cycle marker, revealed a significant number of proliferating cells in the ventricular zone of plxnb2 $2^{+/+}$embryos (Fig. $7 A$, top left, magnified in inset), whereas plxnb2 $2^{-1-}$ embryos only showed a few Ki67-labeled cells within the ventricle (Fig. $7 A$, top right, magnified in inset), but hardly in the ventricular zone. Similarly, the number of proliferating cells was dramatically reduced in the cerebellar EGL of plxnb2 $2^{-1-}$ embryos compared with plxnb2 $2^{+1+}$ embryos, although the choroid plexus showed comparable expression of Ki-67 (Fig. 7A, bottom) $(n=3)$. BrdUlabeling experiments performed in vivo substantiated our observation regarding retarded proliferation and migration of GCPs in the EGL of plxnb2 $2^{-1-}$ embryos (Fig. $7 B)(n=3)$. These results suggest that a defect in proliferation may add on to migratory defects in plxnb2 $2^{-1-}$ embryos, leading to abnormal patterning of the brain.

\section{A putative ligand for Plexin-B2}

Although the expression profile of Sema4D matches that of Plexin-B2 in some brain regions such as the GCP migratory stream of the dentate gyrus, our expression analyses suggest that the developing cerebellum and olfactory bulb represent regions in which Sema4D and Plexin-B2 may not function as a ligandreceptor pair (Worzfeld et al., 2004). Among other class IV semaphorins, Sema4C demonstrates highest expression in cerebellar granule cells in the adult, in which Plexin-B2 is also expressed (data not shown) (Allen Institute for Brain Science; www.brain-map.org/). In a heterologous COS cell system, we observed that a recombinant $\mathrm{AP}$-tagged $\mathrm{N}$-terminal domain of Sema4C (Sema4C-AP) bound Plexin-B2 strongly, but not Plexin-B1 or Plexin-B3 (Fig. 8A,B). Under these conditions, Sema4D-AP strongly bound to Plexin-B1-expressing cells and weakly to those expressing Plexin-B2. It was previously reported that both plexins can function as Sema4D receptors (Masuda et al., 2004). However, by studying binding kinetics with increasing ligand concentrations, we found that Sema4D interacts with Plexin-B2 with a significantly lower affinity (Fig. $8 \mathrm{~B}$ ). The binding of Sema4C to Plexin-B1 was so weak that we could not draw conclusions on the kinetics. In contrast, the $\mathrm{Kd}$ value for Sema4C-Plexin-B2 interaction was calculated to be $0.95 \mathrm{~nm}$ (based on Scatchard plot analysis, 95\% confidence interval between 0.82 and $1.18 \mathrm{nM}$ ).

Moreover, stimulation of HEK293 cells, which naturally express Plexin-B2, with Sema4C-AP led to activation of the Rho GTPase RhoA, as judged via a Rhotekin-based pull-down assay 
A
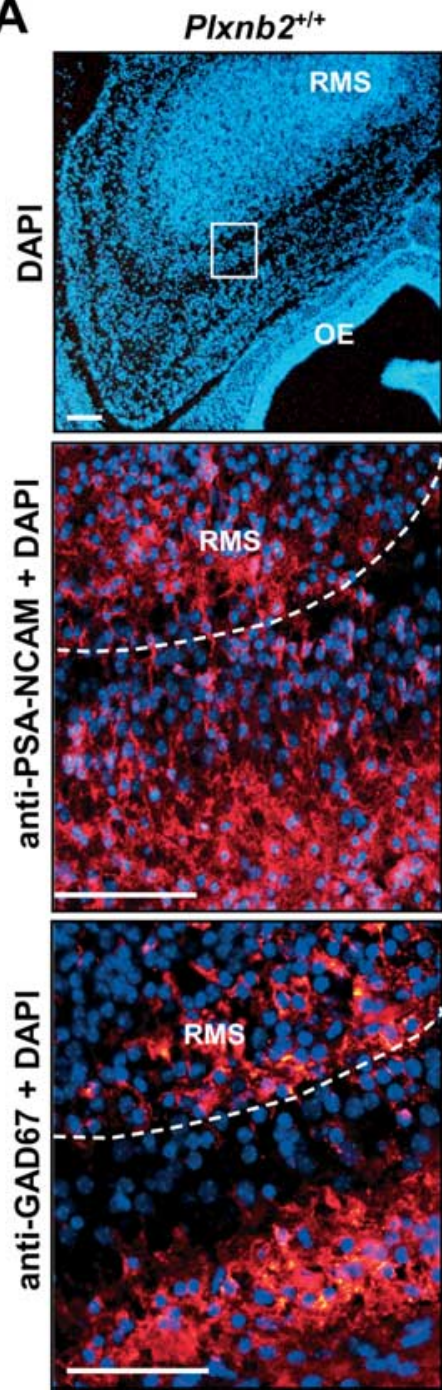

Figure 6. Analysis of olfactory development and proliferation of neuroblasts in plxnb2 embryos. $A, P 1 \times n b 2^{-1-}$ mice that bypass exencephaly show defects in lamination of the olfactory bulb at E18.5 compared with wild-type littermates. Several PSA-NCAM-expressing or GAD67-expressing neuroblasts migrate out of the RMS into olfactory bulb laminas in wild-type mice but are largely retained in RMS of $p / x n b 2^{-1-}$ embryos. Boxed areas are represented at a higher magnification in middle and lower panels. Scale bars, $25 \mu \mathrm{m} . n=3 \mathrm{p} / \mathrm{xnb2} 2^{-1-}$ embryos each with or without exencephaly and equal number of wild-type controls. $0 \mathrm{E}$, Olfactory epithelium.

(Fig. 8C). This effect was enhanced after overexpression of wildtype Plexin-B2 and entirely abolished after overexpression of a C-terminal deletion mutant of Plexin-B2 (Fig. 8C), thereby demonstrating that Sema4C-mediated RhoA activation is mediated via Plexin-B2. Furthermore, Sema4C stimulated phosphorylation of the receptor tyrosine kinase ErbB-2 on Tyr 1248 (Fig. 8C) in a manner similar to Sema4D-induced ErbB-2 phosphorylation via Plexin-B1 activation (Swiercz et al., 2004). Overexpression of Plexin-B2 led to increased phosphorylation of ErbB-2 after Sema4C treatment (Fig. 8C). Consistent with our previous report on the lack of requirement of C-terminal interactions for Sema4D-induced phosphorylation of ErbB-2 via Plexin-B1 (Swiercz et al., 2004), we observed that overexpression of the C-terminal deletion mutant of Plexin-B2 did not reduce Sema4C-induced phosphorylation of ErbB-2. Together, these results indicate that Sema4C can bind Plexin-B2 with a high degree
A
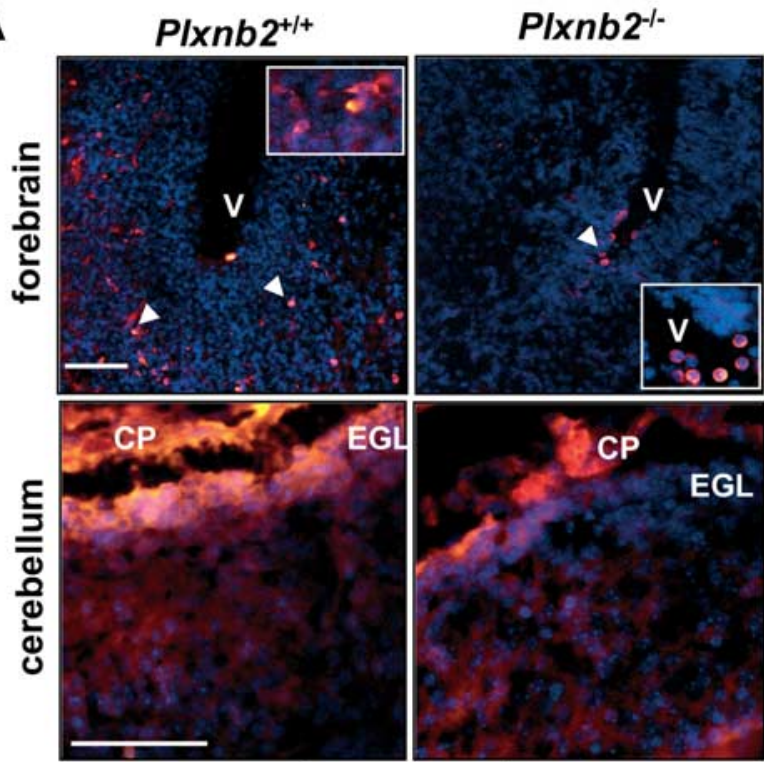

anti-Ki67 + DAPI

B

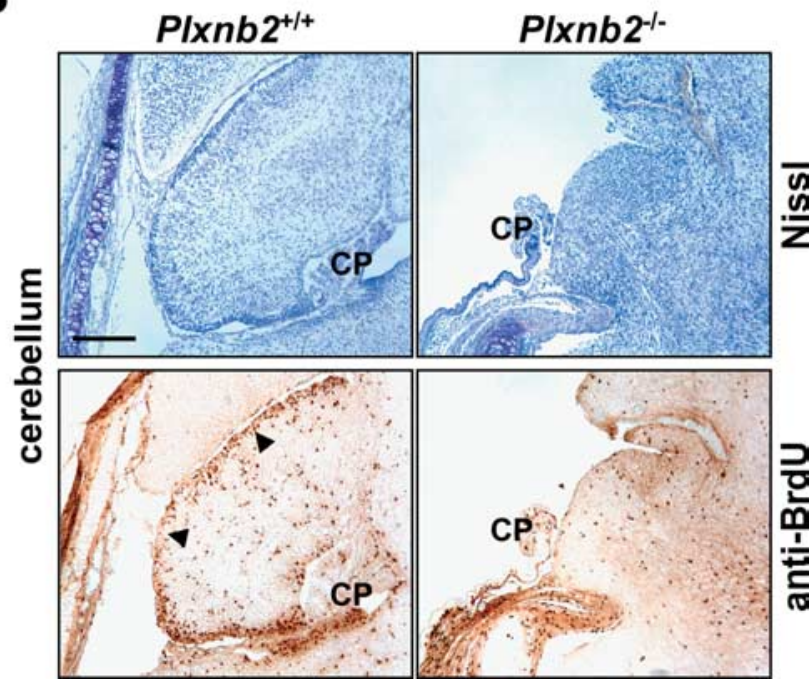

Figure 7. Analysis of defects in proliferation of neuronal precursors in $p / x n b 2^{-1-}$ embryos. $A$, The number of Ki-67-expressing proliferating neuroblasts is strongly reduced in the forebrain ventricular germinative zone (arrowheads) as well as the cerebellar EGL of $p / x n b 2^{-1-}$ mice compared with wild-type littermates at E17.5. Insets show Ki-67-immunoreactive cells in the germinative zone and the ventricles of $p / x n b 2^{+/+}$mice and $p / x n b 2^{-1-}$ mice, respectively. $V$, Ventricle. $\boldsymbol{B}$, In BrdU-labeling experiments at $24 \mathrm{~h}$ after a BrdU pulse given at E16.5, labeled GCPs populate the cerebellar EGL of wild-type littermates (arrowheads), but not in $p / x n b 2^{-1-}$ mice. Top, Nissl-stained adjacent sections. CP, Choroid plexus. Scale bars, $25 \mu \mathrm{m} . n=3$ $p / x n b 2^{-1-}$ embryos each with or without exencephaly and equal number of wild-type controls.

of specificity and high affinity and activate Plexin-B2 leading to RhoA activation and ErbB-2 phosphorylation.

\section{Effects of Sema4C on COS cells expressing Plexin-B2 and} granule cell precursors

Consistent with the above, Sema4C-AP induced a collapse of COS cells expressing Plexin-B2, but not Plexin-B1- or GFPtransfected COS cells (Fig. 9A), suggesting a specific activation of Plexin-B2. These effects were similar to those produced by Sema4D on Plexin-B1-expressing COS cells (Fig. 9) (Tamagnone et al., 1999). 
A

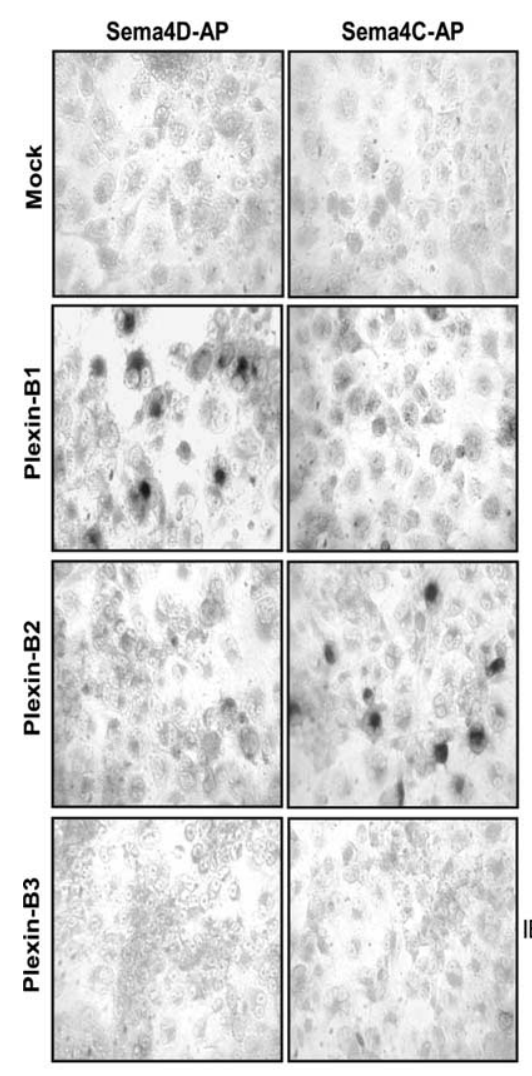

B

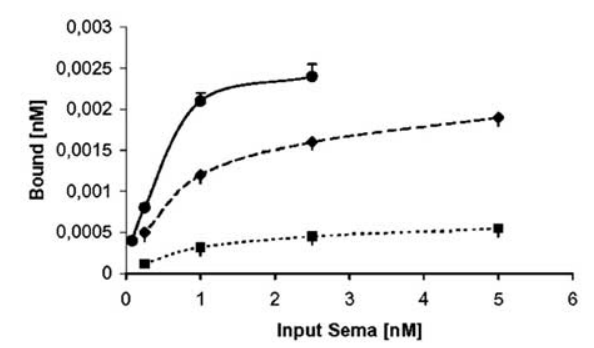

$-\bullet \cdot$ Sema4D on Plexin-B1

$\cdots \cdot \cdot$ Sema4D on Plexin-B2

C
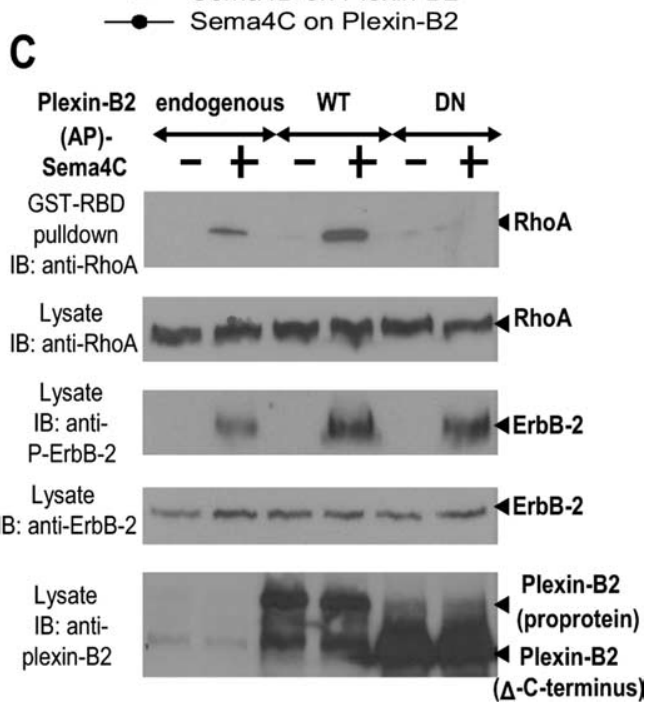

Figure 8. Sema4C binds and activates Plexin-B2.A, Preparations of AP-tagged Sema4D and Sema4C ( $\sim 2$ nm each) specifically bind COS7 cells heterologously expressing Plexin-B1 and Plexin-B2, respectively, but not Plexin-B3, as detected via histochemical detection of AP activity. Expression of plexins was verified via Western blotting. A weak interaction between Plexin-B2-Sema4D and Plexin-B1-Sema4C could be detected after prolonged histochemical reaction. $\boldsymbol{B}$, Binding kinetics as determined by incubating increasing ligand concentrations with Plexin-expressing cells (as above). The amount of bound semaphorins was measured by revealing the associated AP activity with a colorimetric substrate (see Materials and Methods). C, Pull-down assay for activated RhoA showing that treatment of HEK293 cells with Sema4C-AP leads to RhoA activation, which is potentiated after overexpression of Plexin-B2 and abolished after overexpression of a C-terminal deletion mutant of Plexin-B2 ( $\Delta-C$ terminus). In parallel, treatment of HEK293 cells with Sema4C-AP results in phosphorylation of ErbB-2, which is potentiated after overexpression of Plexin-B2 and unaffected by expression of a C-terminal deletion mutant of Plexin-B2. Bottom panels show expression levels of RhoA, endogenous ErbB-2 as well as endogenous and overexpressed Plexin-B2 in cell lysates. WT, Wild type; DN, Dominant negative; IB, immunoblot.

An important question that arises in the light of Plexin-B2 expression in GCPs and the phenotype of Plxnb2 $2^{-1-}$ mice is whether Sema4C can influence functions of GCPs. To address this point, we performed functional assays on GCPs derived from wild-type mice at P5 in a manner similar to that described in Figure 1. GCPs treated with Sema4C-AP medium showed a mild, but statistically significant increase in adhesion to laminin, but not poly-L-lysine or fibronectin, over GCPs treated with equal concentrations of mock-transfection-derived medium (mock; $p<0.05$ for laminin group) (Fig. 9B). Furthermore, Sema4C-AP evoked a significant increase in proliferation of GCPs compared with mock medium ( $p=0.02$ ) (Fig. 9C) (SDF-1 $\alpha$ and epidermal growth factor served as positive controls as mitogens), as judged via BrdU-incorporation experiments. Sema4C-AP stimulated migration of dissociated GCPs in Boyden chamber migration assays ( $p<0.001$ compared with mock medium) (Fig. 9D), demonstrating thereby that Sema4C can modulate properties of developing granule cells. Similarly, Sema4C-AP stimulated the proliferation of dissociated ventricular zone neuroblasts in ex vivo BrdUincorporation assays to an extent similar to that of EGF ( $p<0.001$ compared with mock medium) (Fig. 9E). Importantly, the functional effect of Sema4C, but not of EGF, was entirely lost in mice lacking Plexin-B2 (Fig. 9E), demonstrating thereby that Plexin-B2 is required for this Sema4C-mediated function in developing neurons.

\section{Sema4C expression in the developing cerebellum, dentate gyrus, and olfactory bulb}

Finally, to address whether Sema4C is expressed in a manner consistent with its putative role as a Plexin-B2 ligand, we performed in situ hybridization on the developing brain using a probe that specifically recognizes Sema4c mRNA. At E17.5, Sema4c mRNA was observed to be weakly expressed in the target zone of neuronal precursors migrating via RMS into the developing olfactory bulb (Fig. 10A, arrows), and its expression levels were found to be increased as the olfactory bulb develops over the early postnatal period (an example at P3 is shown in Fig. $10 \mathrm{~B}$ ). The EGL in the developing cerebellum was observed to express Sema4c mRNA moderately at E17.5 (Fig. 10A, arrows) and strongly at P3 (Fig. 10 B, arrows). At P3, some expression was detectable in the IGL (Fig. $10 \mathrm{~B}$ ). Furthermore, Sema4c mRNA is also expressed in the developing dentate gyrus as well as the pyramidal cell layer (the developing CA1 sector, in particular) at E17.5. In contrast to the olfactory bulb and the cerebellum, we observed that the expression of Sema4c in the dentate gyrus decreases after birth (data not shown).

\section{Discussion}

This study was undertaken to understand the functional significance of neuronal plexin-B proteins in the development of the nervous system. The most striking finding of this study is that a loss of Plexin-B2 severely affects the development of the brain in multiple ways, whereas Plexin-B1 deficiency does not significantly impact the development of the CNS and the peripheral nervous system.

The expression of the two neuronal plexin- $\mathrm{B}$ members, Plexin-B1 and Plexin-B2, overlaps in some neural regions during development, e.g., in the germinative neuroepithelium, the cerebellar EGL, and the RMS, but diverges in a striking manner in other regions such as the dentate granule migratory stream, where Plexin-B2 is selectively expressed, or the developing sensory axons of dorsal root ganglia, where Plexin-B1 is selectively expressed (Worzfeld et al., 2004). Therefore, although it is possible that Plexin-B2 can compensate for Plexin-B1 deficiency in the developing cerebellum and olfactory bulb, it is interesting to note that patterning of sensory nerves was not altered in plxnb1 $1^{-1-}$ mice in vivo. The role of Sema4D-Plexin-B1 interactions in sen- 
sory nerve outgrowth had been inconclusive thus far. Although Masuda et al. (2004) reported stimulatory effects of Sema4D on nerve outgrowth, we did not find a functional involvement of PlexinB1-Sema4D in spinal nerve development in vitro (Worzfeld et al., 2004), which was corroborated in the present study in vivo.

One of the most conspicuous consequences of a loss of Plexin-B2 is the failure of cranial neural tube closure, which results in exencephaly and an inversion of the brain. Thus far, there are very few reports implicating plexin-semaphorin pathways in neural tube development. Sema6D, a plexin-A family ligand, and PTK7, a vertebrate homolog of Off-track, a protein kinase that is associated with plexin-A signaling complex, have been linked to neural tube closure (Anderson et al., 2004; Toyofuku et al., 2004). One potential mechanism accounting for the phenotype of $p l x n b 2^{-1-}$ mice may involve modulation of Rho GTPases and thereby of the actin cytoskeleton. Indeed, approaches interfering with RhoA activity, such as inhibition of RhoA-dependent kinase (ROCK) or RhoA knockdown, in neurulating embryos have been reported to result in neural tube defects (Cummings et al., 2001). Interestingly, however, a loss of p190RhoGAP, which is expected to lead to increased Rho activation, is also associated with defects in cranial neural tube closure (Brouns et al., 2000), suggesting that a tight regulation of RhoA activity is required for correct closure of the neural tube. p190RhoGAP is an important member of the plexin-B signaling complex (Barberis et al., 2005). Furthermore, several other regulators of the actin cytoskeleton have been implicated in neural tube closure (Juriloff and Harris, 2000; Wallingford, 2005). It is conceivable therefore that the functional involvement of Plexin-B2 in neural tube closure involves actin organization.

An important aspect of this study is the analysis of the functional role of plexin-B proteins in neuronal migration in vivo. Several studies have reported stimulatory as well as inhibitory modulation of cell migration by class IV semaphorins in vitro (for review, see Kruger et al., 2005). For example, Sema4D-mediated inactivation of R-Ras via the R-RasGAP activity of plexin-B proteins was postulated to inhibit cell migration by suppressing $\beta(1)$-integrin function (Oinuma et al., 2006). In contrast, stimulatory effects of Sema4D on tumor cell migration and invasion have also been reported (Giordano et al., 2002; Basile et al., 2006). However, modulation of neuronal migration by semaphorin-plexin-B interactions had not been explored thus far. Using in vitro assays, we observed that Sema4D stimulated the migration of neuroblasts and GCPs, which were preserved in mice lacking Plexin-B1. The functional contribution and poten- tial compensatory effects of Plexin-B2 to Sema4D-induced promigratory effects could not be directly tested using in vitro assays because of the early mortality of plxnb2 $2^{-1-}$ mutant mice, and it cannot be ruled out that these effects are mediated by nonplexin receptors for Sema4D (Moreau-Fauvarque et al., 2003). Importantly, however, plxnb2 $2^{-1-}$ mice demonstrated striking abnormalities in the migration of GCPs, leading thereby to the defects in lamination and retarded growth of the cerebellum, dentate gyrus, and olfactory bulb. Because plxnb2 $2^{-1-}$ mice develop neural tube defects early, which can lead to nonselective, secondary defects, some caution is warranted in interpreting analysis of migration in plxnb2 $2^{-1-}$ mice in vivo. However, the observations that $p l x n b 2^{-1-}$ mice that do not develop neural tube defects also demonstrate similar migratory defects and that 


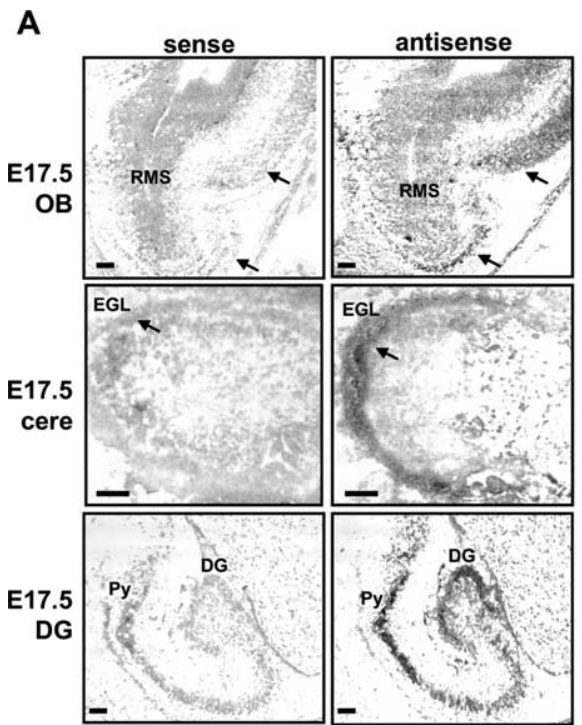

B

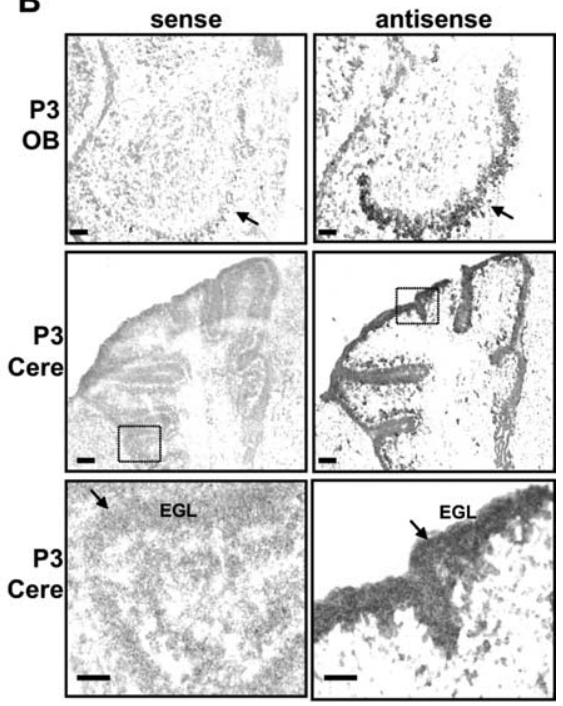

Figure 10. Expression analysis of Sema4c mRNA via in situ hybridization in the developing olfactory bulb (OB), cerebellum (Cere), and dentate gyrus (DG) at E17.5 and P3. Images with sense probes are included in the left panels as controls. $A, B$, Sema $4 C$ mRNA is expressed in target zone of the RMS and in the adjoining cortical plate (arrows) in the developing OB at E17.5 at low levels $(\boldsymbol{A}$, top) and at $\mathrm{P} 3$ at higher levels ( $\boldsymbol{B}$, top). Sema4c is detectable in the EGL of the cerebellum at E17.5 ( $\boldsymbol{A}$, middle, arrows) and at P3 ( $\boldsymbol{B}$, middle and bottom, arrows; the bottom shows a magnified view of the boxed areas in the middle). Sema4c is expressed in developing dentate gyrus (DG) as well as the developing pyramidal cell layer (Py). Scale bars, $25 \mu \mathrm{m}$.

migratory defects are most pronounced in neural regions or cells where Plexin-B2 expression is most intense makes it likely that a loss of Plexin-B2 indeed underlies the observed abnormalities in neuronal migration. Finally, the striking expression pattern and functional requirement for Plexin-B2 in migrating GCPs reported in this study strongly supports the intriguing hypothesis that granule neuron progenitors in the olfactory bulb, hippocampus, and cerebellum share molecular and cellular properties (Yang et al., 1996).

Interestingly, a retardation of GCP migration was also accompanied by defects in cerebellar foliation and by a disorganized patterning of GCPs as well as premigratory granule cells in the EGL, suggesting a role for Plexin-B2 in additional processes involved in the development of the laminar organization of the cerebellum. The plexin-B signaling complex also involves integrins (Barberis et al., 2004; Kruger et al., 2005; Oinuma et al., 2006), and the phenotype mice lacking $\beta$-integrins share striking similarities with that of plxnb2 $2^{-1-}$ mice with respect to cerebellar development (Blaess et al., 2004). Therefore, it is tempting to speculate that changes in integrin function and thereby, in cellmatrix or cell-cell interactions, may underlie these defects, which would be consistent with the perturbation of glial-guided migration of neuronal precursors out of the germinative epithelium observed in the developing hippocampus of plxnb2 $2^{-/-}$mice.

In vivo studies on the role of Sonic hedgehog in cerebellar development have suggested a functional link between cerebellar foliation and GCP proliferation (Dahmane and Ruiz i Altaba, 1999). Here, retarded foliation in $p \ln x b 2^{-1-}$ mice was also accompanied by a strong decrease in GCP proliferation. Interestingly, Met and ErbB-2 are expressed in the developing EGL (Ozaki et al., 1998; Ieraci et al., 2002) and hypomorphic signaling mutants of Met are reported to demonstrate reduced proliferation of GCPs (Ieraci et al., 2002). We also noted that the abundance of actively dividing neuroblasts in germinative ventricular zone was reduced in plxnb2 $2^{-1-}$ mice, suggesting a more wide- spread role for Plexin-B2 in regulating proliferation of neuronal precursors. This may underlie the striking hypotrophy of the neuroepithelium observed in plxnb2 $2^{-1-}$ mutants, regardless of whether or not they developed neural tube defects. Plexin-B2 is indeed expressed in the neuroepithelium, albeit to a lesser degree than Plexin-B1.

Finally, the observation that Plexin-B2 is essential for the development of the cerebellum and the olfactory bulb raises the question about the in vivo ligand for Plexin-B2, given the somewhat incongruous expression pattern of Sema4D. Here, we report that another class IV semaphorin, Sema4C, can bind Plexin-B2 with a high degree of specificity and with high affinity. In contrast, binding kinetics revealed that Plexin-B2 may not be a highaffinity receptor for Sema4D. Although the Kd value for this interaction could not be accurately calculated, we estimated its affinity to be at least five times lower than that of Sema4D for Plexin-B1 (Tamagnone et al., 1999). Here, concentrations of Sema4C as low as $0.5 \mathrm{~nm}$ were found to be sufficient to induce collapse of cells expressing Plexin-B2. Importantly, Sema4C led to ErbB-2 phosphorylation and RhoA activation in a plexin-B2-dependent manner. In vivo, we could detect Sema4c mRNA expression either in those cell populations that express plexin-B2 mRNA or in their close proximity or target zones in the developing olfactory bulb, dentate gyrus, and cerebellum. This suggests that Sema4C-Plexin-B2 may function as a potential ligandreceptor pair in these regions. Consistent with the above, Sema4C stimulated the proliferation and migration of developing GCPs. Importantly, functional assays revealed that proliferative effects of Sema4C are abrogated in mice lacking Plexin-B2, consistent with phenotypic defects in plxnb2 $2^{-1-}$ mice. Together, our expression analysis, the high affinity and specific nature of Sema4C-Plexin-B2 binding, and functional experiments demonstrate that Sema4C-Plexin-B2 can function as a true ligand-receptor pair in vivo.

However, it is important to note that in the context of the plexin-semaphorin system, multiple combinations of ligands and receptor can operate in vivo, an insight that has collectively emerged from a large body of systematic studies on gene knock-outs of class III and class VI semaphorins and plexin-A and -D family members (Takahashi et al., 1999; Cheng et al., 2001; Gu et al., 2005; Suto et al., 2005; Yaron et al., 2005). In analogy, therefore, it is conceivable that diverse semaphorins, such as Sema4D, Sema4C, and potentially others may be able to biologically activate Plexin-B2 at distinct loci in the developing nervous system. Given the strong, early phenotype of classical knock-out mice, future studies involving conditional deletion mutants of Plexin-B2 will be essential to clarify these mechanisms in detail.

Thus, we conclude here that among the neuronal plexin-B proteins, Plexin-B2 is critically required for the patterning of the brain and is a major regulator of neuronal migration and proliferation in vivo. 


\section{References}

Anderson LL, Jeftinija S, Scanes CG (2004) Growth hormone secretion: molecular and cellular mechanisms and in vivo approaches. Exp Biol Med (Maywood) 229:291-302.

Artigiani S, Barberis D, Fazzari P, Longati P, Angelini P, Van De Loo JW, Comoglio PM, Tamagnone L (2003) Functional regulation of semaphorin receptors by proprotein convertases. J Biol Chem 278:10094-10101.

Artigiani S, Conrotto P, Fazzari P, Gilestro GF, Barberis D, Giordano S, Comoglio PM, Tamagnone L (2004) Plexin-B3 is a functional receptor for semaphorin 5A. EMBO Rep 5:710-714.

Aurandt J, Vikis HG, Gutkind JS, Ahn N, Guan KL (2002) The semaphorin receptor plexin-B1 signals through a direct interaction with the Rhospecific nucleotide exchange factor, LARG. Proc Natl Acad Sci USA 99:12085-12090.

Ayoob JC, Terman JR, Kolodkin AL (2006) Drosophila Plexin B is a Sema-2a receptor required for axon guidance. Development 133:2125-2135.

Barberis D, Artigiani S, Casazza A, Corso S, Giordano S, Love CA, Jones EY, Comoglio PM, Tamagnone L (2004) Plexin signaling hampers integrin-based adhesion, leading to Rho-kinase independent cell rounding, and inhibiting lamellipodia extension and cell motility. FASEB J 18:592-594.

Barberis D, Casazza A, Sordella R, Corso S, Artigiani S, Settleman J, Comoglio PM, Tamagnone L (2005) p190 Rho-GTPase activating protein associates with plexins and it is required for semaphorin signalling. J Cell Sci 118:4689-4700.

Basile JR, Castilho RM, Williams VP, Gutkind JS (2006) Semaphorin 4D provides a link between axon guidance processes and tumor-induced angiogenesis. Proc Natl Acad Sci USA 103:9017-9022.

Behar TN, Schaffner AE, Scott CA, O'Connell C, Barker JL (1998) Differential response of cortical plate and ventricular zone cells to GABA as a migration stimulus. J Neurosci 18:6378-6387.

Blaess S, Graus-Porta D, Belvindrah R, Radakovits R, Pons S, LittlewoodEvans A, Senften M, Guo H, Li Y, Miner JH, Reichardt LF, Muller U (2004) Betal-integrins are critical for cerebellar granule cell precursor proliferation. J Neurosci 24:3402-3412.

Brouns MR, Matheson SF, Hu KQ, Delalle I, Caviness VS, Silver J, Bronson RT, Settleman J (2000) The adhesion signaling molecule p190 RhoGAP is required for morphogenetic processes in neural development. Development 127:4891-4903.

Cheng HJ, Bagri A, Yaron A, Stein E, Pleasure SJ, Tessier-Lavigne M (2001) Plexin-A3 mediates semaphorin signaling and regulates the development of hippocampal axonal projections. Neuron 32:249-263.

Conrotto P, Valdembri D, Corso S, Serini G, Tamagnone L, Comoglio PM, Bussolino F, Giordano S (2005) Sema4D induces angiogenesis through Met recruitment by Plexin B1. Blood 105:4321-4329.

Cummings DE, Purnell JQ, Frayo RS, Schmidova K, Wisse BE, Weigle DS (2001) A preprandial rise in plasma ghrelin levels suggests a role in meal initiation in humans. Diabetes 50:1714-1719.

Dahmane N, Ruiz i Altaba A (1999) Sonic hedgehog regulates the growth and patterning of the cerebellum. Development 126:3089-3100.

Fiore R, Puschel AW (2003) The function of semaphorins during nervous system development. Front Biosci 8:s484-s499.

Giordano S, Corso S, Conrotto P, Artigiani S, Gilestro G, Barberis D, Tamagnone L, Comoglio PM (2002) The semaphorin 4D receptor controls invasive growth by coupling with Met. Nat Cell Biol 4:720-724.

Govek EE, Newey SE, Van Aelst L (2005) The role of the Rho GTPases in neuronal development. Genes Dev 19:1-49.

Gu C, Yoshida Y, Livet J, Reimert DV, Mann F, Merte J, Henderson CE, Jessell TM, Kolodkin AL, Ginty DD (2005) Semaphorin 3E and plexin-D1 control vascular pattern independently of neuropilins. Science 307:265-268.

Guan KL, Rao Y (2003) Signalling mechanisms mediating neuronal responses to guidance cues. Nat Rev Neurosci 4:941-956.

Hack I, Bancila M, Loulier K, Carroll P, Cremer H (2002) Reelin is a detachment signal in tangential chain-migration during postnatal neurogenesis. Nat Neurosci 5:939-945.

Hirotani M, Ohoka Y, Yamamoto T, Nirasawa H, Furuyama T, Kogo M, Matsuya T, Inagaki S (2002) Interaction of plexin-B1 with PDZ domain-containing Rho guanine nucleotide exchange factors. Biochem Biophys Res Commun 297:32-37.
Hu H, Marton TF, Goodman CS (2001) Plexin B mediates axon guidance in Drosophila by simultaneously inhibiting active Rac and enhancing RhoA signaling. Neuron 32:39-51.

Ieraci A, Forni PE, Ponzetto C (2002) Viable hypomorphic signaling mutant of the Met receptor reveals a role for hepatocyte growth factor in postnatal cerebellar development. Proc Natl Acad Sci USA 99:15200-15205.

Juriloff DM, Harris MJ (2000) Mouse models for neural tube closure defects. Hum Mol Genet 9:993-1000.

Klein RS, Rubin JB, Gibson HD, DeHaan EN, Alvarez-Hernandez X, Segal RA, Luster AD (2001) SDF-1 alpha induces chemotaxis and enhances Sonic hedgehog-induced proliferation of cerebellar granule cells. Development 128:1971-1981.

Kruger RP, Aurandt J, Guan KL (2005) Semaphorins command cells to move. Nat Rev Mol Cell Biol 6:789-800.

Lakso M, Pichel JG, Gorman JR, Sauer B, Okamoto Y, Lee E, Alt FW, Westphal H (1996) Efficient in vivo manipulation of mouse genomic sequences at the zygote stage. Proc Natl Acad Sci USA 93:5860-5865.

Liu BP, Strittmatter SM (2001) Semaphorin-mediated axonal guidance via Rho-related G proteins. Curr Opin Cell Biol 13:619-626.

Masuda K, Furuyama T, Takahara M, Fujioka S, Kurinami H, Inagaki S (2004) Sema4D stimulates axonal outgrowth of embryonic DRG sensory neurones. Genes Cells 9:821-829.

Moreau-Fauvarque C, Kumanogoh A, Camand E, Jaillard C, Barbin G, Boquet I, Love C, Jones EY, Kikutani H, Lubetzki C, Dusart I, Chedotal A (2003) The transmembrane semaphorin Sema4D/CD100, an inhibitor of axonal growth, is expressed on oligodendrocytes and upregulated after CNS lesion. J Neurosci 23:9229-9239.

Nagata I, Nakatsuji N (1990) Granule cell behavior on laminin in cerebellar microexplant cultures. Brain Res Dev Brain Res 52:63-73.

Negishi M, Oinuma I, Katoh H (2005) Plexins: axon guidance and signal transduction. Cell Mol Life Sci 62:1363-1371.

Neufeld G, Shraga-Heled N, Lange T, Guttmann-Raviv N, Herzog Y, Kessler O (2005) Semaphorins in cancer. Front Biosci 10:751-760.

Oinuma I, Ishikawa Y, Katoh H, Negishi M (2004) The Semaphorin 4D receptor Plexin-B1 is a GTPase activating protein for R-Ras. Science 305:862-865.

Oinuma I, Katoh H, Negishi M (2006) Semaphorin 4D/Plexin-B1-mediated R-Ras GAP activity inhibits cell migration by regulating beta(1) integrin activity. J Cell Biol 173:601-613.

Ozaki M, Kishigami S, Yano R (1998) Expression of receptors for neuregulins, ErbB2, ErbB3 and ErbB4, in developing mouse cerebellum. Neurosci Res 30:351-354.

Perrot V, Vazquez-Prado J, Gutkind JS (2002) Plexin B regulates Rho through the guanine nucleotide exchange factors leukemiaassociated Rho GEF (LARG) and PDZ-RhoGEF. J Biol Chem 277:43115-43120.

Raper JA (2000) Semaphorins and their receptors in vertebrates and invertebrates. Curr Opin Neurobiol 10:88-94.

Ren XD, Schwartz MA (2000) Determination of GTP loading on Rho. Methods Enzymol 325:264-272.

Suto F, Ito K, Uemura M, Shimizu M, Shinkawa Y, Sanbo M, Shinoda T, Tsuboi M, Takashima S, Yagi T, Fujisawa H (2005) Plexin-a4 mediates axon-repulsive activities of both secreted and transmembrane semaphorins and plays roles in nerve fiber guidance. J Neurosci 25:3628-3637.

Swiercz JM, Kuner R, Behrens J, Offermanns S (2002) Plexin-B1 directly interacts with PDZ-RhoGEF/LARG to regulate RhoA and growth cone morphology. Neuron 35:51-63.

Swiercz JM, Kuner R, Offermanns S (2004) Plexin-B1/RhoGEF-mediated RhoA activation involves the receptor tyrosine kinase ErbB-2. J Cell Biol 165:869-880.

Takahashi T, Fournier A, Nakamura F, Wang LH, Murakami Y, Kalb RG, Fujisawa H, Strittmatter SM (1999) Plexin-neuropilin-1 complexes form functional semaphorin-3A receptors. Cell 99:59-69.

Tamagnone L, Artigiani S, Chen H, He Z, Ming GI, Song H, Chedotal A, Winberg ML, Goodman CS, Poo M, Tessier-Lavigne M, Comoglio PM (1999) Plexins are a large family of receptors for transmembrane, secreted, and GPI-anchored semaphorins in vertebrates. Cell 99:71-80.

Toyofuku T, Zhang H, Kumanogoh A, Takegahara N, Suto F, Kamei J, Aoki 
K, Yabuki M, Hori M, Fujisawa H, Kikutani H (2004) Dual roles of Sema6D in cardiac morphogenesis through region-specific association of its receptor, Plexin-A1, with off-track and vascular endothelial growth factor receptor type 2. Genes Dev 18:435-447.

Vikis HG, Li W, He Z, Guan KL (2000) The semaphorin receptor plexin-B1 specifically interacts with active Rac in a ligand-dependent manner. Proc Natl Acad Sci USA 97:12457-12462.

Wallingford JB (2005) Neural tube closure and neural tube defects: studies in animal models reveal known knowns and known unknowns. Am J Med Genet C Semin Med Genet 135:59-68.

Worzfeld T, Puschel AW, Offermanns S, Kuner R (2004) Plexin-B family members demonstrate non-redundant expression patterns in the de- veloping mouse nervous system: an anatomical basis for morphogenetic effects of Sema4D during development. Eur J Neurosci 19:2622-2632.

Yang XW, Zhong R, Heintz N (1996) Granule cell specification in the developing mouse brain as defined by expression of the zinc finger transcription factor RU49. Development 122:555-566.

Yaron A, Huang PH, Cheng HJ, Tessier-Lavigne M (2005) Differential requirement for Plexin-A3 and -A4 in mediating responses of sensory and sympathetic neurons to distinct class 3 Semaphorins. Neuron 45:513-523.

Yuasa S (2001) Development of astrocytes in the mouse hippocampus as tracked by tenascin-C gene expression. Arch Histol Cytol 64:149-158. 